\title{
Magnetism and structure of magnetic multilayers based on the fully spin polarized Heusler alloys $\mathrm{Co}_{2} \mathrm{MnGe}$ and $\mathrm{Co}_{2} \mathrm{MnSn}$
}

\author{
K. Westerholt, A. Bergmann, J. Grabis, A. Nefedov and H. Zabel
}

October 19, 2018

Institut für Experimentalphysik IV, Ruhr-Universität Bochum, D-44780

Bochum, Germany

\begin{abstract}
Our Introduction starts with a short general review of the magnetic and structural properties of the Heusler compounds which are under discussion in this book. Then, more specifically, we come to the discussion of our experimental results on multilayers composed of the Heusler alloys $\mathrm{Co}_{2} \mathrm{MnGe}$ and $\mathrm{Co}_{2} \mathrm{MnSn}$ with $\mathrm{V}$ or $\mathrm{Au}$ as interlayers. The experimental methods we apply combine magnetization and magneto-resistivity measurements, x-ray diffraction and reflectivity, soft x-ray magnetic circular dichroism and spin polarized neutron reflectivity. We find that below a critical thickness of the Heusler layers at typically $d_{c r}=1.5 \mathrm{~nm}$ the ferromagnetic order is lost and spin glass order occurs instead. For very thin ferromagnetic Heusler layers there are peculiarities in the magnetic order which are unusual when compared to conventional ferromagnetic transition metal multilayer systems. In $\left[\mathrm{Co}_{2} \mathrm{MnGe} / \mathrm{Au}\right]$ multilayers there is an exchange bias shift at the ferromagnetic hysteresis loops at low temperatures caused by spin glass ordering at the interface. In $\left[\mathrm{Co}_{2} \mathrm{MnGe} / \mathrm{V}\right]$ multilayers we observe an antiferromagnetic interlayer long range ordering below a well defined Néel temperature originating from the dipolar stray fields at the magnetically rough Heusler layer interfaces. Copy of a book article edited by P.H. Dederichs and J. Galanakis "Halfmetallic Alloys - Fundamentals and Applications" Lecture Notes in Physics Springer, p67-110 (2005)
\end{abstract}

\section{Introduction}

In the main part of this article we will describe our experimental results on thin films and multilayers based on the Heusler phases $\mathrm{Co}_{2} \mathrm{MnGe}$ and $\mathrm{Co}_{2} \mathrm{MnSn}$. However, before coming to this special topic, we will give a short review of the 
structural and magnetic properties of the Heusler compounds in general with special emphasis on the half metallic Heusler compounds. This is intended to give a general introduction to the experimental chapters of this book, where the focus of the investigations is on thin films of Heusler phases with theoretically predicted half metallic character. A knowledge of the metallurgical, structural and magnetic properties of bulk Heusler compounds will help the reader to estimate the problems one encounters when trying to grow thin films of the Heusler compounds optimized for spintronic applications.

The Heusler compounds have a long history in magnetism, starting more than 100 years ago with the detection of the ternary metallic compound $\mathrm{Cu}_{2} \mathrm{MnAl}$ by A. Heusler [1]. That time the most interesting point with this phase was that it represented the first transition metal compound with ferromagnetically ordered Mn-spins. Later it turned out that a whole class of isostructural ternary metallic alloys with the general composition $\mathrm{X}_{2} \mathrm{YZ}$ exists, where $\mathrm{X}$ denotes a transition metal element such as $\mathrm{Ni}, \mathrm{Co}, \mathrm{Fe}$ or $\mathrm{Pt}, \mathrm{Y}$ is a second transition metal element, e.g. $\mathrm{Mn}, \mathrm{Cr}$ or $\mathrm{Ti}$ and $\mathrm{Z}$ is an atom from $3^{\text {rd }}, 4^{\text {th }}$ or $5^{\text {th }}$ group of the periodic system such as $\mathrm{Al}, \mathrm{Ge}, \mathrm{Sn}$ or Sb. More than 1000 different Heusler compounds have been synthesized until now, a comprehensive review of the experimental work until the year 1987 can be found in [2].

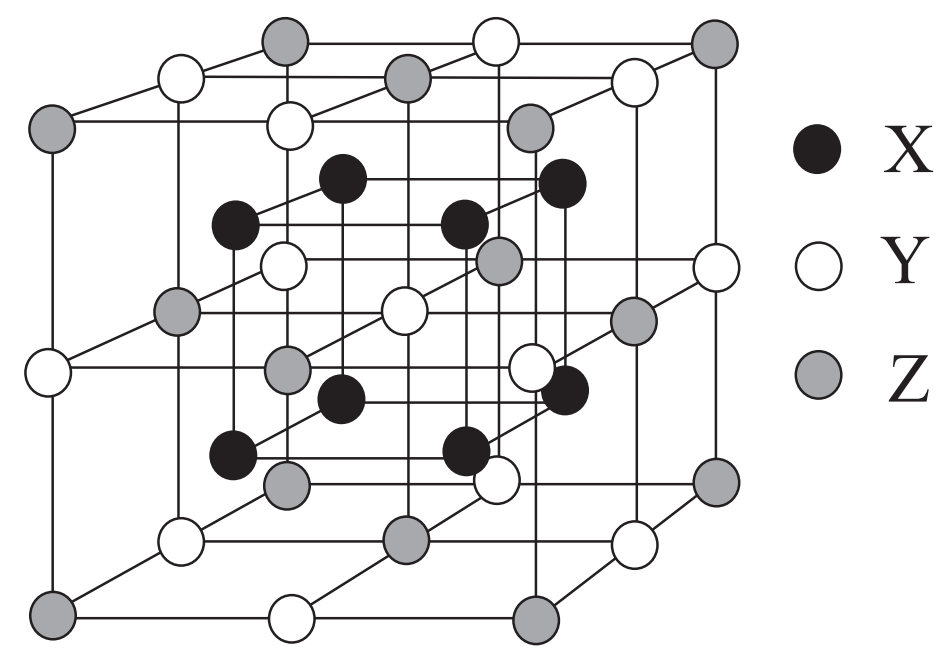

\section{L2 ${ }_{1}\left(\mathrm{X}_{2} \mathrm{YZ}\right)$}

Figure 1: Schematic representation of the L $2{ }_{1}$-structure.

The original Heusler phase $\mathrm{Cu}_{2} \mathrm{MnAl}$, which later gave its name to the whole class of ternary inter-metallic compounds, can be considered as the prototype of all other Heusler compounds, including those with half metallic properties. It has been studied intensively over the past 100 years and we begin with describ- 
ing its properties in some detail. The crystal structure of $\mathrm{Cu}_{2} \mathrm{MnAl}$ is cubic with three different structural modifications: At high temperatures the crystal structure is bcc with random occupation of the $\mathrm{Cu}, \mathrm{Mn}$ and $\mathrm{Al}$ atoms on the lattice sites of a simple bcc lattice with a lattice parameter $a=0.297 \mathrm{~nm}$. At low temperatures the equilibrium crystal structure is fcc, space group L $2_{1}$, with the lattice parameter doubled to $a=0.595 \mathrm{~nm}$ compared to the bcc high temperature phase. The $\mathrm{L} 2{ }_{1}$-structure is most important for the predicted half metallic properties of the Heusler compounds discussed in this book, since the theoretical band structure calculations usually refer to this structure. The L $2_{1}$ unit cell is depicted in Fig 1 and can be imagined to be combined of four interpenetrating fcc-sublattices occupied by $\mathrm{Mn}, \mathrm{Al}$ and $\mathrm{Cu}$ atoms respectively and shifted along the space diagonal with the corner of the Al-sublattice at $(0,0,0)$, the first $\mathrm{Cu}$-sublattice at $(1 / 4,1 / 4,1 / 4), \mathrm{Mn}$ at $(1 / 2,1 / 2,1 / 2)$ and the second $\mathrm{Cu}$-sublattice at $(3 / 4,3 / 4,3 / 4)$. There exists a third intermediate crystal structure for the $\mathrm{Cu}_{2} \mathrm{MnAl}$ compound having $\mathrm{B} 2$-symmetry. It has the same lattice parameter as the $\mathrm{L} 2{ }_{1}$ phase, but in the unit cell depicted in Fig. 1 the $\mathrm{Mn}$ - and $\mathrm{Al}$ - atoms are distributed at random on the $\mathrm{Mn}$ - and Al-sublattices whereas the two $\mathrm{Cu}$-sublattices remain intact.

In $\mathrm{Cu}_{2} \mathrm{MnAl}$ the $\mathrm{B} 2$ and the $\mathrm{L} 21_{1}$ phases develop from the high temperature bcc structure by chemical ordering of the atoms on the 4 fcc-sublattices in two steps via two second order structural phase transitions at $1040 \mathrm{~K}$ (bcc-B2) and $900 \mathrm{~K}\left(\mathrm{~B} 2-\mathrm{L} 2_{1}\right)$ [3]. The structural order parameter of these transitions can be calculated by measuring the intensity of the superstructure Bragg reflection (200) for the bcc-B2 transition or the Bragg reflection (111) for the B2-L2 2 -transition. Since in these order-disorder phase transitions diffusion of atoms is involved, long time annealing or slow cooling is necessary to achieve complete chemical ordering. In the majority of the Heusler compounds these or similar structural phase transitions exist, they can be hysteretic and of first order or reversible and of second order [2]. For several Heusler phases there in addition exist low temperature structural distortions of the cubic unit cell, the most prominent example being $\mathrm{Ni}_{2} \mathrm{MnGa}$ with a martensitic phase transition at $175 \mathrm{~K}$. $\mathrm{Ni}_{2} \mathrm{MnGa}$ is the only ferromagnetic transition metal alloy which exhibits shape memory properties [4]. For $\mathrm{Cu}_{2} \mathrm{MnAl}$ and other Heusler phases one often encounters problems with the metallurgical stability. Below $T=900 \mathrm{~K}$ the $\mathrm{Cu}_{2} \mathrm{MnAl}$ phase tends to decompose into $\beta-\mathrm{Mn}, \mathrm{Cu}_{9} \mathrm{Al}_{4}$ and $\mathrm{Cu}_{3} \mathrm{AlMn}_{2}$ 5. Experimentally one can overcome this problem by suppressing the nucleation of the secondary phases by rapid quenching the Heusler alloys from high temperatures.

The magnetism of the Heusler alloys is very versatile and has been under continuous discussion all over the past hundred years. The majority of the Heusler alloys with a magnetic element on the Y-position order ferromagnetically, but several antiferromagnetic compounds also exists, $\mathrm{Ni}_{2} \mathrm{MnAl}$ or $\mathrm{Pd}_{2} \mathrm{MnAl}$ provide examples [6, 7]. The main contribution to the magnetic moments in the Heusler phases usually stems from the atoms at the Y-position. If magnetic atoms also occupy the X-positions, their moment is usually quite small or even vanishing. In the above mentioned $\mathrm{Ni}_{2} \mathrm{MnAl}$ compound e.g. the Ni-atoms are non-magnetic. 
Actually there exist a few Heusler compounds with rather large magnetic moments on both the X-and the Y-positions. In this case the ferromagnetic state is very stable and the ferromagnetic Curie temperatures $T_{c}$ become exceptionally high. The best examples are provided by the Heusler phases $\mathrm{Co}_{2} \mathrm{MnSi}$ and $\mathrm{Co}_{2} \mathrm{FeSi}$ with a Co-moment of about $1 \mu \mathrm{B}$ and Curie temperatures of $985 \mathrm{~K}$ [8] and $1100 \mathrm{~K} \mathrm{[9],} \mathrm{respectively,} \mathrm{the} \mathrm{highest} T_{c^{-}}$-values known for the Heusler alloys. The reason for this very stable ferromagnetism is a strong next nearest neighbor ferromagnetic exchange interaction between the spins at the $\mathrm{X}$ - and the $\mathrm{Y}$-position. If a non-magnetic element occupies the $\mathrm{X}$-position, the dominant exchange interaction between the Y-spins is of weaker superexchange type, mediated by the electrons of the non-magnetic Z- and X-atoms [10].

Heusler compounds such as $\mathrm{Cu}_{2} \mathrm{MnAl}$ with a magnetic moment only on the Y-position are considered as good examples of localized $3 d$ metallic magnetism 10. Since in the ideal $\mathrm{L} 2{ }_{1}$ structure there are no $\mathrm{Mn}-\mathrm{Mn}$ nearest neighbours, the Mn $3 d$-wave functions overlap only weakly and the magnetic moments remain essentially localized at the Mn-position. However, in the family of Heusler compounds there also exist beautiful examples of weak itinerant ferromagnetism with strongly delocalized magnetic moments. The compound $\mathrm{Co}_{2} \mathrm{TiSn}$ with magnetic moments only on the X-positions belongs to this category [11]. As it is obvious from the crystallographic structure (Fig 1), there are nearest neighbor X-atoms making the overlap of the $3 d$-wave-functions and the delocalized character of the $d$-electrons much larger than in the case of only the atoms at the Y-position being magnetic. Replacing the $\mathrm{Co}$-atoms in $\mathrm{Co}_{2} \mathrm{TiSn}$ by $\mathrm{Ni}$, this delocalization effect proceeds further, making the compound $\mathrm{Ni}_{2} \mathrm{TiSn}$ a Pauli paramagnet [12]. Even more interesting, the Heusler compounds $\mathrm{Fe}_{2} \mathrm{TiSn}$ and $\mathrm{Fe}_{2} \mathrm{VAl}$ also do not order magnetically, but are marginally magnetic. They belong to the rare class of transition metal compounds exhibiting heavy Fermion like properties in the low temperature specific heat and the electrical resistivity, which attracted much interest in the literature in recent years [13, 14, 15].

Now, coming to the fully spin polarized Heusler compounds which are of main interest in the present book, the first Heusler compounds with a gap in the electron density of states for the spin down electron band at the Fermi level were detected in 1983 by electron energy band structure calculations [16]. The first two compounds with this spectacular property were NiMnSb and PtMnSb, so called semi Heusler alloys (space group $\mathrm{C}_{b}$ ) where in the Heusler unit cell of Fig 1 one of the X-sublattices remains empty. The semi Heusler structure only exists if the Z-position is occupied by atoms with large atomic radii such as Sn or Sb. The NiMnSb and PtMnSb compounds have been dubbed half metals [16, since only for one spin direction there is metallic conductivity, for the other spin direction the conductivity is of semiconducting type. In a ferromagnetic transition metal alloy this half metallicity is a very rare property, since usually $s$ - or $p$-bands with a small exchange splitting cross the Fermi energy and contribute states of both spin directions. For several years PtMnSb and NiMnSb remained the only ferromagnetic alloys with half metallic character, before starting from 1990 a second group of half metallic Heusler alloys, $\mathrm{Co}_{2} \mathrm{MnSi}, \mathrm{Co}_{2} \mathrm{MnGe}$ and $\mathrm{Co}_{2} \mathrm{Mn}\left(\mathrm{Sb}_{x} \mathrm{Sn}_{1-x}\right)$ was detected [17, 18, 19]. 
The experimental proof of half metallicity in these Heusler alloys is a long and still ongoing controversial issue. The first attempts to prove the half metallicity used electron transport measurement to test the existence of a gap in the spin down electron band [20, 21. Since in the half metal for temperatures small compared to the gap in the minority spin band there is only one spin direction at the Fermi level available, it is expected that electronic scattering processes involving spin flips and longitudinal spin wave excitations are inhibited. Thus one should expect an increasing electron mobility and a change of the power law describing the temperature dependence of the resistivity when the temperature becomes smaller than the gap for the minority spin band. Actually for NiMnSb this type of behavior for temperatures below $80 \mathrm{~K}$ has been detected, additionally in this temperature range the Hall coefficient exhibits an anomalous temperature dependence, strongly suggesting a thermal excitation of charge carriers across a gap coexisting with metallic conductivity [20, 21]. More directly, positron annihilation experiments on bulk single crystals from the NiMnSb phase were found to be consistent with $100 \%$ spin polarization at the Fermi level [22. Finally, analyzing the current-voltage characteristic below the superconducting gap of a point contact between a Nb-superconductor and a bulk PtMnSb sample, which is dominated by Andreev reflections at the ferromagnet/superconductor interface, the authors derived a spin polarization of $90 \%$ at the Fermi level [23].

For the $\mathrm{Co}_{2} \mathrm{Mn}(\mathrm{Si}, \mathrm{Ge}, \mathrm{Sn})$ fully spin polarized group of Heusler compounds spin polarized neutron diffraction measurements on bulk samples have been employed to determine the degree of spin polarization at the Fermi level [24]. This methods probe the spatial distribution of the magnetization, details of which depend sensitively on the spin polarization. The main conclusion is that the spin polarization is large but not $100 \%$. More recent superconducting/ferromagnetic point contact measurements on a $\mathrm{Co}_{2} \mathrm{MnSi}$ single crystal gave a spin polarization of $55 \%$ 25. Similarly, the degree of spin polarization determined from the analysis of spin resolved photoemission spectra was always found to be definitely below $100 \%$ [26, 27].

During the first years after the discovery of the half metallic character in the Heusler compounds they were considered as exotic ferromagnets of mainly academic interest. This attitude has changed completely with the development of new ideas of data storage and processing designed to use both, the charge and the spin degree of freedom of the conduction electrons, nowadays called spin electronics (spintronics) [28, 29]. In the spintronic community there is a strong belief that in the future these new concepts have the perspective to complement or even substitute conventional Si technology. It was rapidly realized how valuable it would be for spintronic devices to have a ferromagnet available with only one conduction electron spin direction at the Fermi level. With an electrode possessing $100 \%$ spin polarization the generation of a fully spin polarized current for spin injection into semiconductors would be very easy [30], spin filtering and spin accumulation in metallic thin film systems would be most effective [31] and the tunneling magnetoresistance (TMR) [32] of a device prepared of two half metallic electrodes should have a huge magneto-resistance, since the tunneling probability is vanishing to first order if the two electrodes 
have an antiparallel magnetization direction.

Thus the novel concepts of spintronics started an upsurge of interest in ferromagnetic half metals in the literature. Principally there are three different classes of ferromagnetic half metals which are under intense discussion in the literature and regarded as possible candidates to be used in spintronic devices. These three groups combine semiconductors such as $\mathrm{GaAs}$ or $\mathrm{ZnO}$ doped by magnetic transition metal ions [33], magnetic oxides such as $\mathrm{CrO}_{2}, \mathrm{Fe}_{3} \mathrm{O}_{4}$ or $\left(\mathrm{Sr}_{1-x} \mathrm{La}_{x}\right) \mathrm{MnO}_{3}[34,35]$ and, last but not least, the fully spin polarized Heusler compounds under discussion in this book.

Actually with each of these three essentially different groups of materials one encounters serious problems when thinking of possible applications. The question which group or which compound is the best is completely open. The magnetically doped semiconductors all share the problem of magnetic inhomogeneity and rather low ferromagnetic Curie temperatures, making them unsuitable for room temperature applications. For the ferromagnetic oxides, on the other hand, the main problem is that the preparation and handling of these compounds is completely incompatible with current semiconductor technology. In this respect the fully spin polarized Heusler compounds appear to be the better choice, since they can be prepared by conventional methods of metallic thin film preparation. A further advantage of the Heusler compounds, especially in comparison with the magnetically doped semiconductors, is their high ferromagnetic Curie temperature. However, as already stated above and frequently discussed in the following chapters of this book, the half metallicity of the Heusler compounds is a subtle property which is easily lost in a real sample.

Recent intense work by theoretical groups, several of them represented in this book, increased the number of Heusler compounds with predicted half metallic properties to more than 20, among them $\mathrm{Rh}_{2} \mathrm{MnGe}, \mathrm{Fe}_{2} \mathrm{MnSi}$ and $\mathrm{Co}_{2} \mathrm{CrAl}$, to mention a few of the new compounds [36, 37, 38, 39, 40]. From the experimental side, most of these phases have not been studied thoroughly yet, thus leaving a vast field for future experimental investigations. The experimental work in the literature mainly concentrated on the classical Heusler half-metallic phases $\mathrm{NiMnSb}$ and $\mathrm{PtMnSb}$, the alloys $\mathrm{Co}_{2} \mathrm{MnSi}$ and $\mathrm{Co}_{2} \mathrm{MnGe}$ and the newly discovered compound $\mathrm{Co}_{2}(\mathrm{CrFe}) \mathrm{Al}$ [38].

When thinking of possible applications in spintronic devices, which at the moment is the main motivation behind the experimental research on the fully spin polarized Heusler compounds in the literature, it is obvious that one needs thin films of the Heusler compounds, bulk samples are not very useful. This is the reason why in the majority of the experimental chapters in this book thin film systems are studied. Additional detailed research on high quality bulk material, however, is highly welcome and urgently needed for purposes of comparison with the thin films and in order to elucidate the basic properties of the compounds. The recent XAFS and neutron scattering study of $\mathrm{Co}_{2} \mathrm{MnSi}$ single crystals provides a beautiful example [41, 42]. It allowed a precise determination of the number antisite defects in the crystal (Co sitting on the Mn position and vice versa), which would be very difficult in thin films, but is important for a realistic judgment of the perspective to reach full spin polarization in a 
$\mathrm{Co}_{2} \mathrm{MnSi}$ thin films prepared under optimum conditions.

In the experimental chapters of this book it will become clear to the reader that using the predicted full spin polarization of the Heusler compounds in thin film structures is difficult and the technical development is still in its infancy. Thin film preparation in general and especially the preparation of thin film heterostructures, often imposes limits on the process parameters and this might severely interfere with the needs to have of a high degree of spin polarization. For obtaining a large spin polarization a perfect crystal structure with a small number of grain boundaries is important. This can best be achieved by keeping the substrate at high temperatures during the thin film deposition. However, most Heusler phases grow in the Vollmer-Weber mode (three-dimensional islands) at high temperatures, thus when using high preparation temperatures there might be a strong roughening of the surfaces which for spintronic devices is strictly prohibited. In addition, in thin film heterostructures combining different metallic, semiconducting or insulating layers with the Heusler compounds, high preparation temperatures are forbidden, since excessive interdiffusion at the interfaces must be avoided.

Interfaces of the Heusler compounds with other materials are a very delicate problem for spintronic devices. For spin injection into semiconductors or a tunneling magnetoresistance the spin polarization of the first few monolayers at the interfaces is of utmost importance. A large spin polarization in the bulk of a Heusler compound does not guarantee that it is a good spintronic material, unless it keeps its spin polarization down to the first few monolayers at the interfaces. Thin films and TMR-devices have first been investigated systematically using the semi Heusler compounds PtMnSb and NiMnSb 42. The performance was rather disappointing e.g. the maximum value for the TMR achieved was of the order of $12 \%$ only. Later, TMR devices based on $\mathrm{Co}_{2} \mathrm{MnSi}$ was shown to work much better [44] (see article by J. Schmalhorst in this book), but still the calculated spin polarization is definitely smaller than $100 \%$.

This experience leads to the suspicion that at least for a few monolayers at the interfaces the full spin polarization is somehow lost. One possible explanation for this could be modifications in the electronic energy band structure at surfaces and interfaces, since the theoretical predictions of a full spin polarization in a strict sense only hold for bulk crystals. Recent band structure calculations taking the surfaces explicitly into account revealed that for certain crystallographic directions the full spin polarization at the surfaces is actually lost [45, 46]. Even more seriously, it seems that atomic disorder of the Heusler compound at surfaces and interfaces has the tendency to severely affect the full spin polarization. Theoretical band structure calculations predicting the half metallicity assume L $2{ }_{1}$-symmetry with perfect site symmetry of all four X, Y and $\mathrm{Z}$ sublattices. As mentioned above, the existence of some antisite disorder, however, cannot be completely avoided even in well annealed single crystals [41, 42].

An essential question is, which type of disorder is most detrimental for the spin polarization of the Heusler compounds. Model calculations taking different 
types of point defects into account are very illustrative in this respect [47, 39] (see also the article by S. Picocci in this book). These calculations show that for the $\mathrm{Co}_{2} \mathrm{MnGe}$ and the $\mathrm{Co}_{2} \mathrm{MnSi}$ Heusler phase the electronic states of Co-antisite atoms sitting on regular Mn-sites fill the gap in the minority spin band. The magnetic moment of these Co antisite defect remains virtually unchanged and couples ferromagnetically to the surrounding spins. A Mn-antisite atom sitting on a regular Co-position, on the other hand, does not introduce electronic states in the minority spin gap, however has its magnetic moment coupled antiferromagnetically to the surrounding spins. This leads to a drastic reduction of the saturation magnetization, which frequently has been observed for ferromagnetic Heusler phases not prepared under optimum conditions.

Introducing now the present article which reviews our work during the last four years on thin films and multilayers based on the Heusler alloys $\mathrm{Co}_{2} \mathrm{MnGe}$ and $\mathrm{Co}_{2} \mathrm{MnSn}$, our main interest was not on spintronic devices for the moment, although we will present examples of GMR devices in chapter IV. We, instead, go one step backwards and study first the basic structural and magnetic properties of very thin films of these Heusler compounds. The magnetism of a ternary metallic compound is complex and little is known of what happens with the magnetic order in the limit of very thin films or at interfaces. Then we present our results on the magnetic order in multilayers of the Heusler alloys using $\mathrm{V}$ and $\mathrm{Au}$ as interlayers, which can be grown with high quality. Neutron and x-ray scattering provide well established methods to get insight into the structural and magnetic properties of the interfaces. And, by the way, we follow the interesting and until now unsolved question, whether an oscillating interlayer exchange coupling (IEC) between the Heusler layers can be observed when varying the thickness of the $\mathrm{V}$ - and Au-layers systematically.

In detail, our article below is organized as follows: In Sec. 2 we discuss the magnetic and structural properties of single films of the $\mathrm{Co}_{2} \mathrm{MnGe}$ and $\mathrm{Co}_{2} \mathrm{MnSn}$ phase for a thickness range down to about $3 \mathrm{~nm}$. In Sec. 3 we discuss the properties of multilayers prepared by combining these Heusler layers with $\mathrm{Au}$ and $\mathrm{V}$ interlayers. In this section we also review in some detail a novel type of antiferromagnetic interlayer long range order which we have observed in $\left[\mathrm{Co}_{2} \mathrm{MnGe} / \mathrm{V}\right]_{n}$ multilayers and an unidirectioal exchange anisotropy originating from the interface magnetic order in the $\left[\mathrm{Co}_{2} \mathrm{MnGe} / \mathrm{Au}\right]_{n}$ multilayers. In Sec. 4 we present results of our resistivity and magnetoresistivity measurements on the single films, multilayers and GMR devices, before we summarize and draw conclusions in Sec. 5 ,

\section{Single $\mathrm{Co}_{2} \mathrm{MnGe}$ and $\mathrm{Co}_{2} \mathrm{MnSn}$ thin films}

\subsection{Structural properties}

All films of the present study were grown by rf-sputtering with a dual source $\mathrm{HV}$ sputtering equipment on single crystalline $\mathrm{Al}_{2} \mathrm{O}_{3}$ (11-20) surfaces (sapphire a-plane). The base pressure of the system was $5 \times 10^{-8}$ mbar after cooling of 
the liquid nitrogen cold trap. We used pure Ar at a pressure of $5 \times 10^{-3}$ mbar as sputter gas, the targets were prepared from stoichiometric alloys of the Heusler phases. The sputtering rates during the thin film preparation were $0.06 \mathrm{~nm} / \mathrm{s}$ for the $\mathrm{Co}_{2} \mathrm{MnSn}$ phase and $0.04 \mathrm{~nm} / \mathrm{s}$ for the $\mathrm{Co}_{2} \mathrm{MnGe}$ phase, the $\mathrm{Au}$ or $\mathrm{V}$ seed layers were deposited at a sputtering rate of 0.05 and $0.03 \mathrm{~nm} / \mathrm{s}$ respectively. The sapphire substrates were cleaned chemically and ultrasonically after cutting and immediately before the deposition they were additionally etched by an ion beam for $300 \mathrm{~s}$ in order to remove any residual surface contaminations. There are two conditions to achieve good structural and magnetic quality of the Heusler layers. First, the substrate temperature must be high, in our optimized procedure the substrate temperature was $500^{\circ} \mathrm{C}$. Second, seed layers of a simple metal with a good lattice matching to the Heusler compounds are important to induce epitaxial or textured growth with a flat surface. Growing the films directly on sapphire results in polycrystalline films and surface roughening. In our previous investigations we tested different possible seed layers such as $\mathrm{Cr}$, $\mathrm{Nb}$ and $\mathrm{Ag}$, later we concentrated on $\mathrm{Au}$ and $\mathrm{V}$ which gave the best structural results [67, 66]. The surfaces of the Heusler compounds oxidize rapidly and must be covered by a protection layer before handling in air. If nothing else is stated we use an amorphous $\mathrm{Al}_{2} \mathrm{O}_{3}$ film of 2-nm thickness as a cap layer.

\begin{tabular}{|c|c|c|c|c|c|}
\hline phase & \multicolumn{2}{|c|}{ lattice parameter $[\mathrm{nm}]$} & \multicolumn{2}{|c|}{ sat. magnetization $\left[\frac{\mathrm{emu}}{\mathrm{g}}\right]$} & sat. moment $\left[\mu_{B}\right]$ \\
\hline & bulk & film & bulk & film & film \\
\hline $\mathrm{Co}_{2} \mathrm{MnGe}$ & 0.5743 & 0.575 & 111 & 114 & 5.02 \\
$\mathrm{Co}_{2} \mathrm{MnSn}$ & 0.600 & 0.6001 & 91 & 92 & 4.95 \\
\hline
\end{tabular}

Table 1: Lattice parameters, saturation magnetization and saturation magnetic moments (per formula unit) of the pure, thick Heusler films in comparison to the bulk values. The bulk values have been taken from reference [2].

The structural quality of the samples was studied by small angle $\mathrm{x}$-ray reflectivity and high angle out-of-plane Bragg scattering using $\mathrm{Cu} K_{\alpha}$ radiation. Figure 2 show an out of plane x-ray Bragg scan of a $\mathrm{Co}_{2} \mathrm{MnSn}$ film with a 2$\mathrm{nm}$ thick $\mathrm{Au}$ seed layer and $\mathrm{Co}_{2} \mathrm{MnGe}$ film with a 5 -nm V seed layer. Only the (220) and the (440) Bragg peaks of the Heusler phases are observed, proving perfect (110) texture out-of-plane. In plane the films are polycrystalline. Table 1 summarizes the relevant structural data derived from the x-ray scans. The lattice parameters virtually coincide with those of the bulk phases. The half width of the rocking curve (width in $2 \Theta$ at half maximum) characterizing the mosaicity of the crystallite is $1.5^{\circ}$ for the $\mathrm{Co}_{2} \mathrm{MnSn}$ phase and $0.8^{\circ}$ for the $\mathrm{Co}_{2} \mathrm{MnGe}$ phase. The half width of the radial Bragg scans corresponds to the experimental resolution of the spectrometer implying that the total thickness of the Heusler layer is structurally coherent in the growth direction. Examples of a small angle x-ray reflectivity scan for films of the same phases are shown in figure 3. Total thickness oscillations are observed up to $2 \theta=10^{\circ}$, indicatiove of 

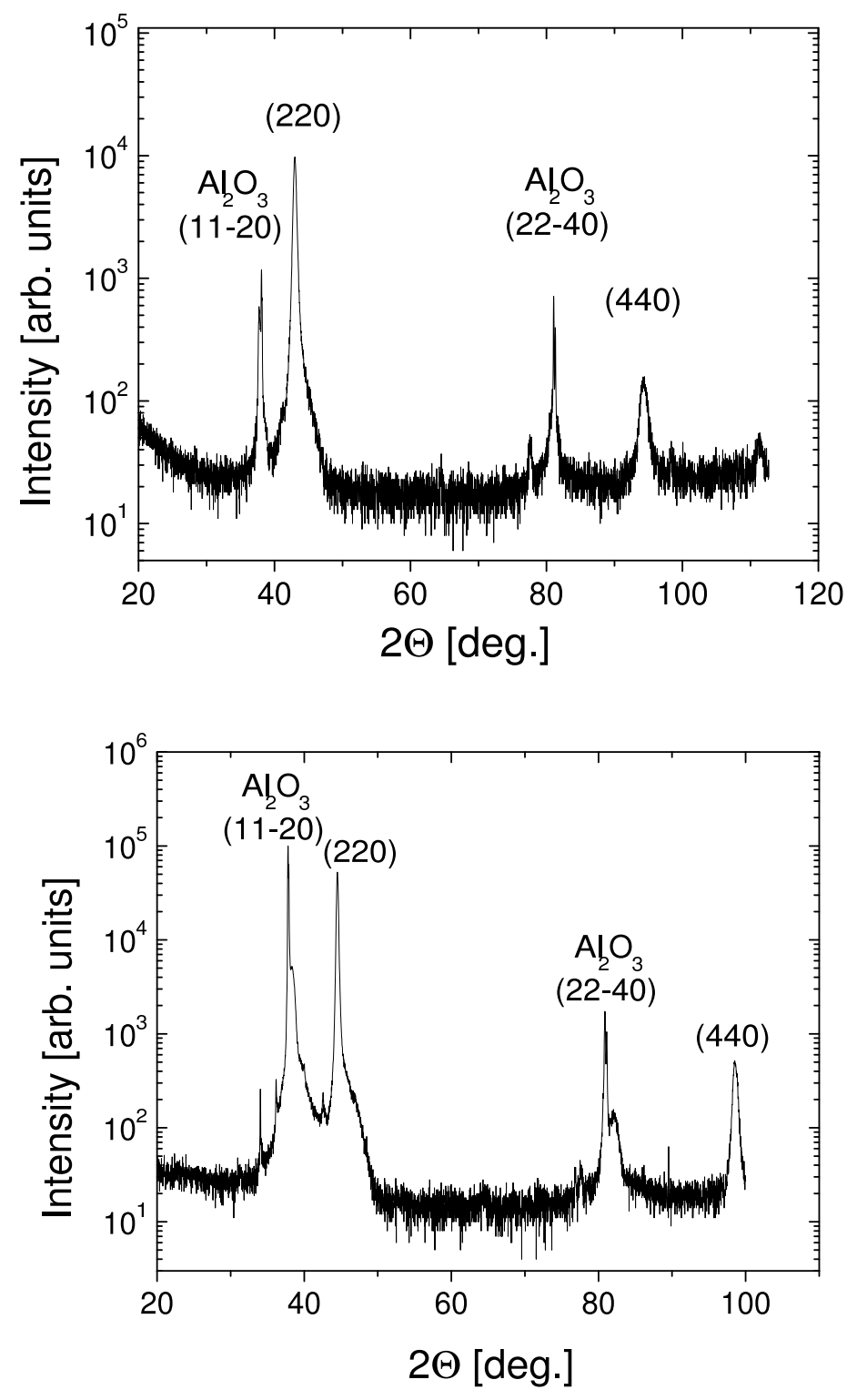

Figure 2: Upper panel (a): Out of plane Bragg scans of a $\mathrm{Au}(3 \mathrm{~nm}) / \mathrm{Co}_{2} \mathrm{MnSn}(100 \mathrm{~nm})$ film measured with $\mathrm{Cu} \mathrm{K} \mathrm{K}_{\alpha}$ radiation. Bottom panel (b): same for a $\mathrm{V}(3 \mathrm{~nm}) / \mathrm{Co}_{2} \mathrm{MnGe}(100 \mathrm{~nm})$ film. 
a very flat surface morphology. additional oscillations from the buffer layer are also observed. A simulation of the curves with the Parratt formalism gives a typical rms roughness for the film surface of about $0.3 \mathrm{~nm}$. This is corroborated by atomic force microscopic (AFM) pictures of the surface which reveal a very smooth surface morphology. We also have grown films at lower preparation temperatures down to a substrate temperature $T_{\text {sub }}=100^{\circ} \mathrm{C}$. The structure of films prepared below $T_{s}=500^{\circ} \mathrm{C}$ is still perfect (110) texture, however with a rocking width slightly increasing with decreasing preparation temperature.

Since for the multilayers discussed in the next chapter the typical layer thickness of the Heusler layers is of the order of $3 \mathrm{~nm}$ only and $T_{s}$ is limited to $300^{\circ} \mathrm{C}$ in order to avoid strong interdiffusion at the interfaces, it seems worthwhile to study the structural and magnetic properties of single very thin Heusler layers prepared under the same conditions. Figure 4 shows an out-of- plane Bragg scan of a trilayer $\mathrm{V}(5 \mathrm{~nm}) / \mathrm{Co}_{2} \mathrm{MnGe}(4 \mathrm{~nm}) / \mathrm{V}(5 \mathrm{~nm})$. One observes a very broad (220) Bragg peak with a half width of $\Delta 2 \Theta=2^{\circ}$ at $2 \Theta=43^{\circ}$ from the $\mathrm{Co}_{2} \mathrm{MnGe}$ phase. Using the Scherrer formula for the Bragg reflection of small particles, which correlates the coherence length $L_{c}$ of the particles with the half width of the Bragg peaks

$$
L_{c}=0.89 \lambda /[\Delta(2 \Theta) \cdot \cos (\Theta)]
$$

we derive $L_{c}=3 \mathrm{~nm}$ i.e. a value slightly smaller than the layer thickness. This is an indication that the mean size of the crystalline grains in the film is slightly smaller than the film thickness. For $\mathrm{Co}_{2} \mathrm{MnGe}$ layers of 4-nm thickness between two Au layers, which we also have studied, the grain size was found to be definitely larger than the film thickness, since the correlation length is only limited by the finite film thickness.

\subsection{Magnetic properties}

The dc magnetization of our films was studied by a commercial superconducting quantum interference device (SQUID) based magnetometer (Quantum Design MPMS system). Examples of magnetic hysteresis loops of the $\mathrm{Co}_{2} \mathrm{MnGe}$ and the $\mathrm{Co}_{2} \mathrm{MnSn}$ films are presented in figure 5 . The films possess a growth induced weak, uniaxial anisotropy with an anisotropy field $H_{K}$ of about 50 Oe. For the measurements in figures 5 and 6 the external field axis was directed parallel to the magnetic easy axis, thus the hysteresis loops are rectangular. The coercive field for the $\mathrm{Co}_{2} \mathrm{MnGe}$ phase is $H_{c}=20 \mathrm{Oe}$, for the $\mathrm{Co}_{2} \mathrm{MnSn}$ film we get $H_{c}=50 \mathrm{Oe}$ at room temperature. The saturation magnetization at $5 \mathrm{~K}$ is in good agreement with the values measured in bulk samples within the experimental error bars (see table 1). The saturation magnetic moment per formula unit calculated from the saturation magnetization is $4.95 \mu_{B}$ for $\mathrm{Co}_{2} \mathrm{MnSn}$ and $5.02 \mu_{B}$ for $\mathrm{Co}_{2} \mathrm{MnGe}$. These values agree with those derived from the theoretical band structure calculations for perfect L2 $2_{1}$ type of order [36], indicating that the films have a high degree of metallurgical order. Thus 

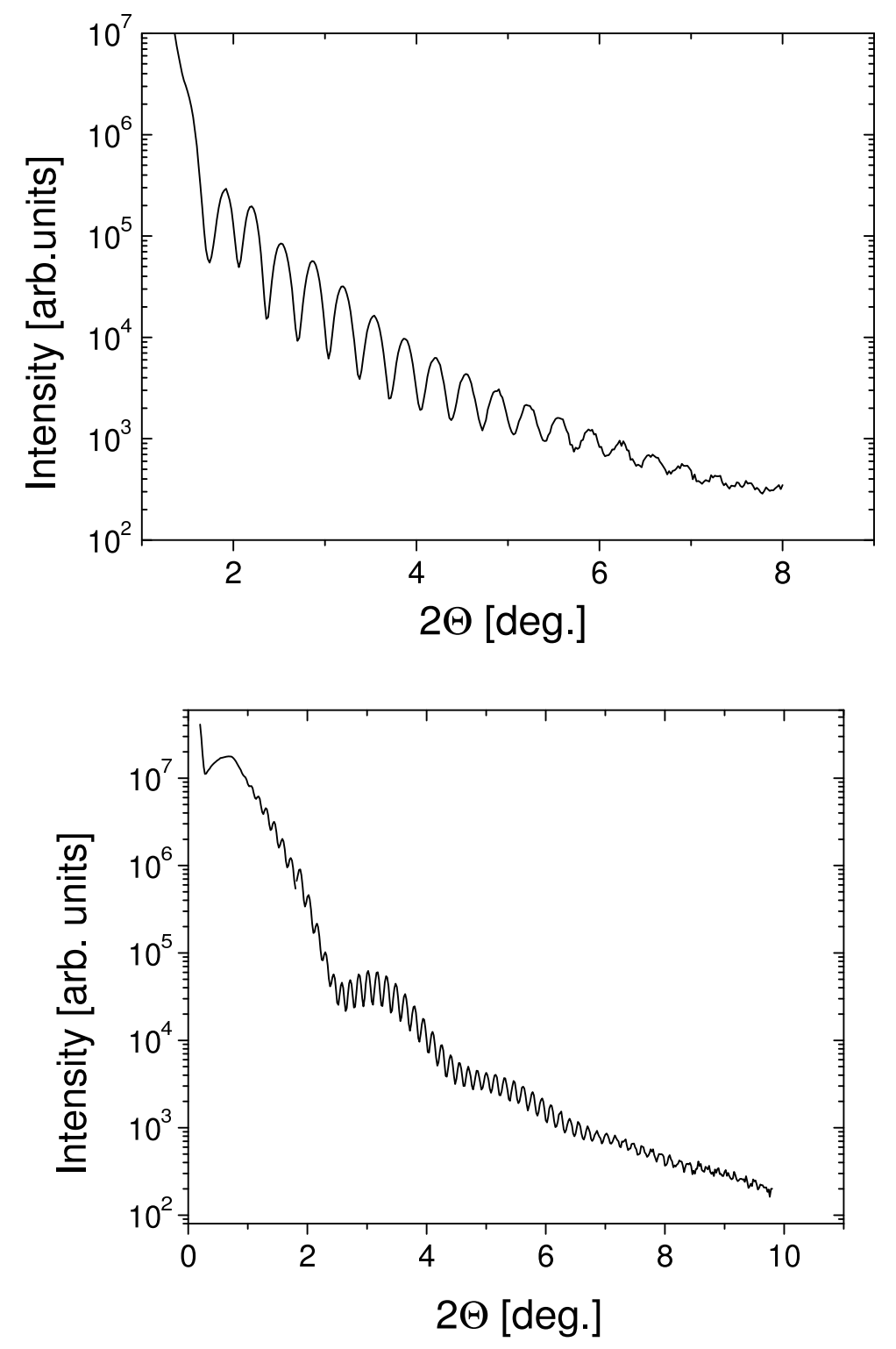

Figure 3: X-ray reflectivity scans of $\mathrm{Au}(3 \mathrm{~nm}) / \mathrm{Co}_{2} \mathrm{MnSn}(30 \mathrm{~nm})$ in the upper panel (a) and $\mathrm{V}(4 \mathrm{~nm}) / \mathrm{Co}_{2} \mathrm{MnGe}(60 \mathrm{~nm})$ in the bottom panel (b). 


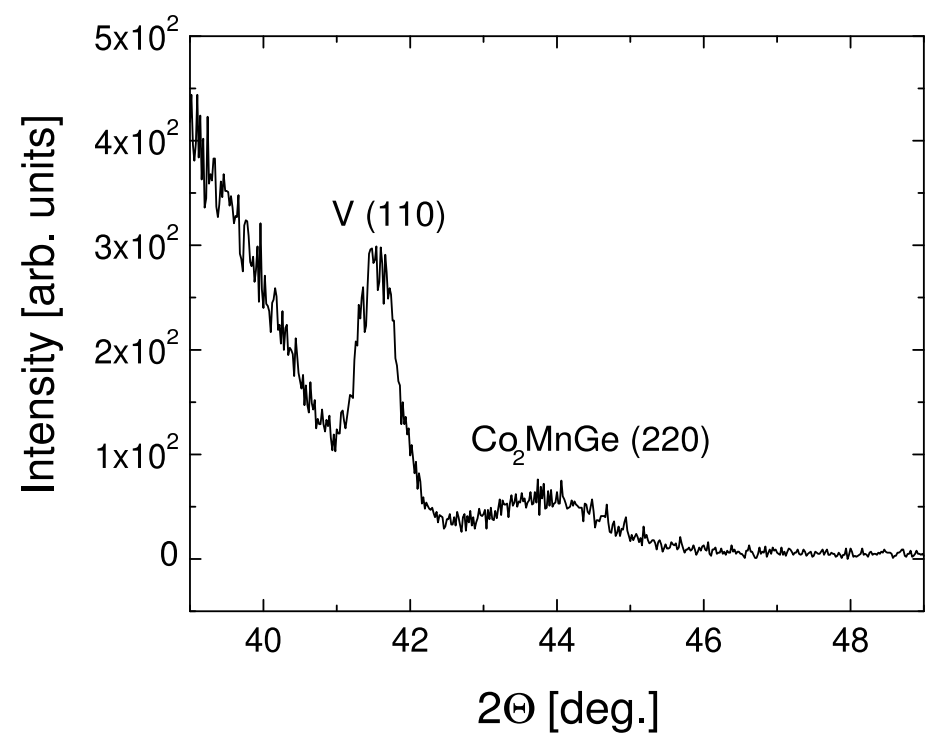

Figure 4: Out of plane Bragg scan of a $\mathrm{V}(5 \mathrm{~nm}) / \mathrm{Co}_{2} \mathrm{MnGe}(4 \mathrm{~nm}) / \mathrm{V}(5 \mathrm{~nm})$ trilayer.

we can conclude that thick films of the Co based Heusler phases can be grown at high temperatures with a quality comparable to bulk samples.

However, when decreasing the substrate temperature while keeping the thickness of the $\mathrm{Co}_{2} \mathrm{MnGe}$ layer and all other parameters constant, we observe a continuous decrease of the saturation magnetization down to about $70 \%$ at $T_{s}=100^{\circ} \mathrm{C}$ (figure 6). This decrease of the magnetization is accompanied by an increase of the lattice parameter of about $1 \%$. For the $\mathrm{Co}_{2} \mathrm{MnSn}$ phase and $\mathrm{Cu}_{2} \mathrm{MnAl}$ the degradation of the ferromagnetic saturation magnetization with decreasing $T_{s}$ is even faster (figure 6). It seems plausible to attribute the decrease of the saturation magnetization to an increasing number of antisite defects in the $\mathrm{L} 22_{1}$ structure. This effect is well known in $\mathrm{Cu}_{2} \mathrm{MnAl}$, where the disordered B2 phase which can be prepared by quenching from high temperatures has a very low saturation magnetization [73]. For films with a small thickness of the Heusler phase of the order of a few $\mathrm{nm}$ the situation becomes even worse. In this case a preparation at $500^{\circ} \mathrm{C}$ is prohibited, since then interdiffusion at the seed layer/Heusler interface is too strong. A practical limit for the substrate temperature for avoiding excessive interdiffusion is $300^{\circ} \mathrm{C}$. As a first example of a very thin film, figure 7 depicts the hysteresis loop measured at $5 \mathrm{~K}$ of a 4-nm thick $\mathrm{Co}_{2} \mathrm{MnGe}$ film grown directly on sapphire a-plane at $T_{s}=300^{\circ} \mathrm{C}$. The x-ray structural analysis showed no resolvable Bragg peak, thus the crystalline structure seems to be polycrystalline with very small grains. The film has a very low saturation magnetization of only about $10 \%$ of the bulk value, 

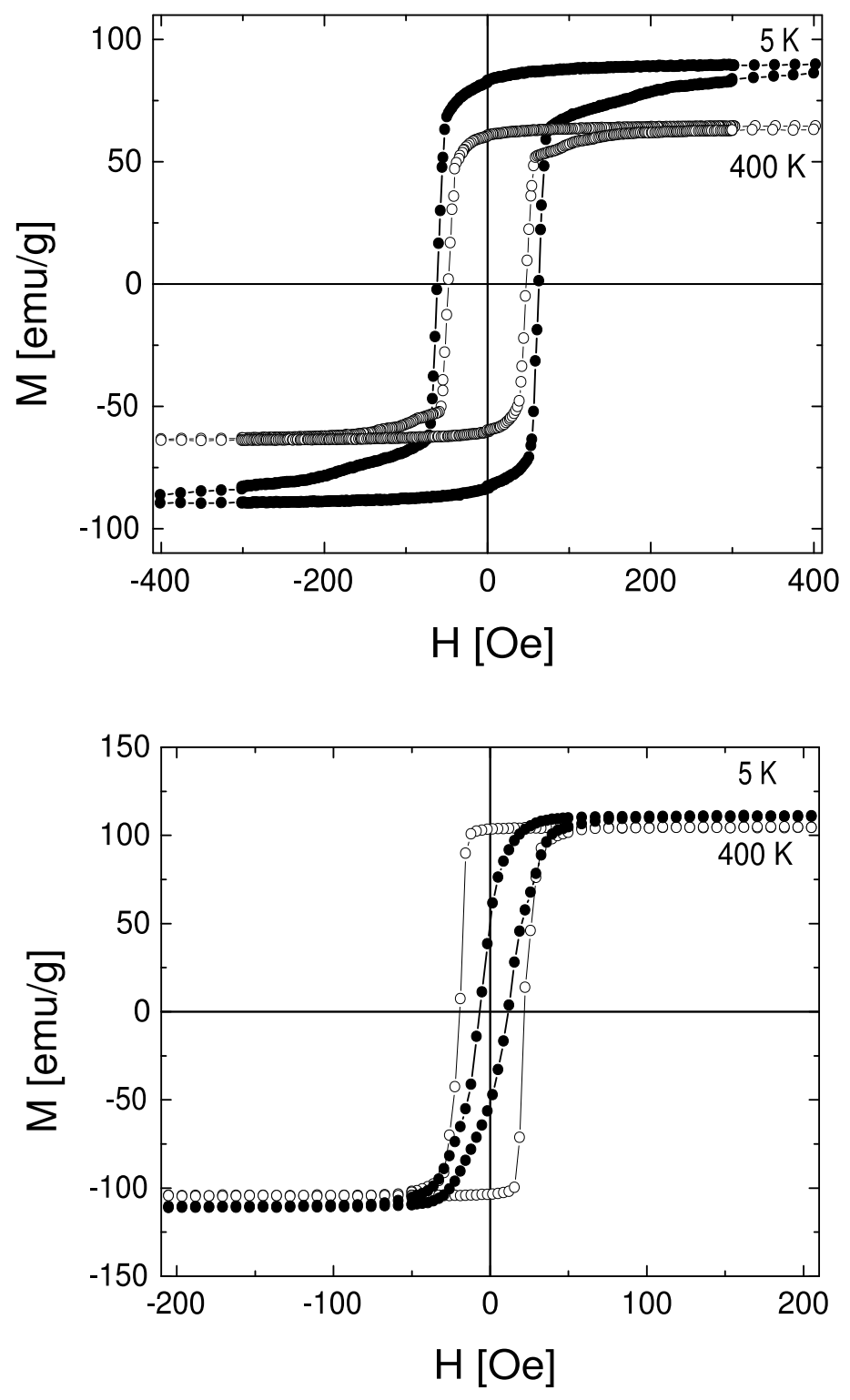

Figure 5: Hysteresis loops of a $\mathrm{Co}_{2} \mathrm{MnSn}$ in the upper panel (a) and a $\mathrm{Co}_{2} \mathrm{MnGe}$ film in the bottom panel (b) from figure 2 measured at $5 \mathrm{~K}$ and $400 \mathrm{~K}$. 


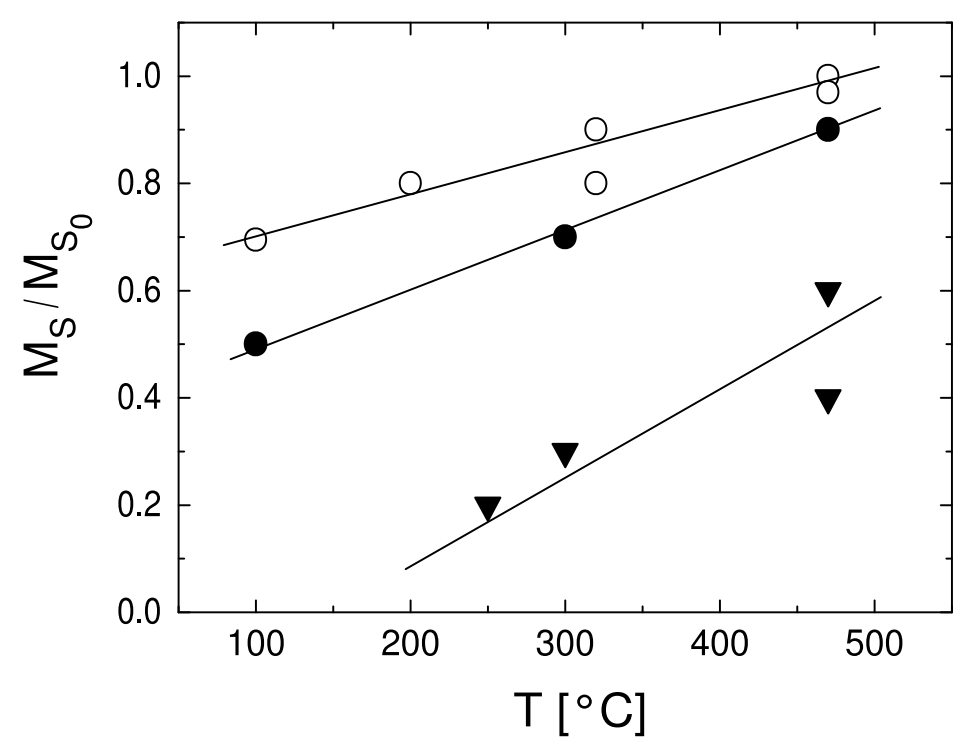

Figure 6: Saturation magnetization of $\mathrm{Co}_{2} \mathrm{MnGe}$ (open circles), $\mathrm{Co}_{2} \mathrm{MnSn}$ (solid circles) and $\mathrm{Cu}_{2} \mathrm{MnAl}$ (triangles) versus the substrate temperature during preparation.

showing that the ferromagnetic properties are completely different from those of the $\mathrm{Co}_{2} \mathrm{MnGe}$ phase in the $\mathrm{L} 2_{1}$ structure. Growing the same film thickness for $\mathrm{Co}_{2} \mathrm{MnGe}$ on a V seedlayer, a (220) Bragg peak can be observed (see figure 4) and about $50 \%$ of the ferromagnetic saturation magnetization of the $\mathrm{Co}_{2} \mathrm{MnGe}$ phase is recovered (figure 8). The magnetization is isotropic in the film plane with a magnetic remanence of only about $30 \%$ of the saturation magnetization.

It is interesting to note that for a field direction perpendicular to the film plane (figure $8 \mathrm{~b}$ ) the hysteresis curves are very similar to those observed in a parallel field, thus the strong anisotropy of the demagnetising field characterizing a homogeneous magnetic thin film is absent. This clearly shows that the magnetization of the film in figure 8 is not homogeneous, but breaks up into weakly coupled small magnetic particles pointing in their own magnetically easy direction given by the geometric shape and the crystal magnetic anisotropy. For a 4-nm thick $\mathrm{Co}_{2} \mathrm{MnGe}$ film grown on $\mathrm{Au}$ the small particle character of the hysteresis loop also exists, but the magnetic anisotropy of a homogenous thin film is partly recovered. For this film the ferromagnetic saturation magnetization is also strongly reduced compared to the bulk value of $\mathrm{Co}_{2} \mathrm{MnGe}$. We would attribute the different magnetic behaviour of $\mathrm{Co}_{2} \mathrm{MnGe}$ grown on $\mathrm{V}$ and $\mathrm{Au}$ to much smaller crystalline grains in the case of a $\mathrm{V}$ seed layer, where the single grains seem to be nearly decoupled magnetically. 


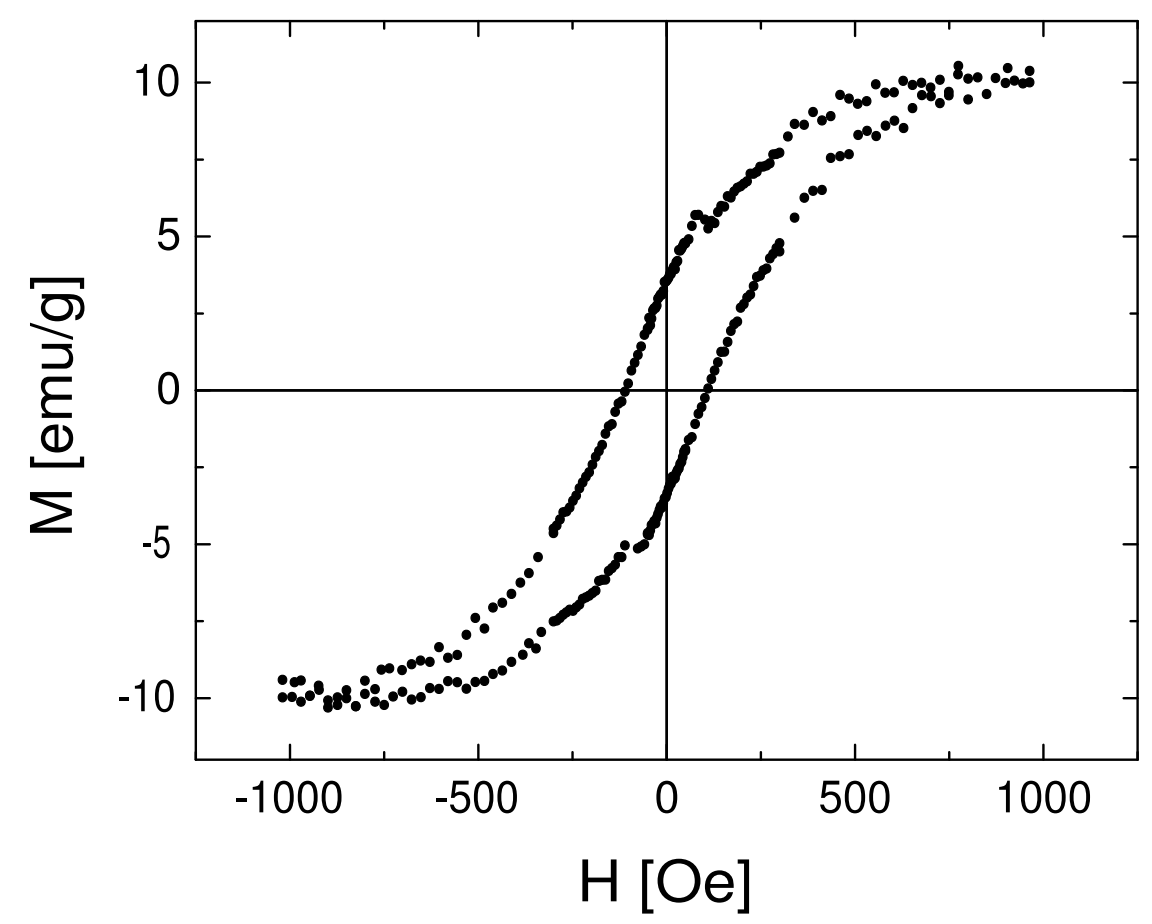

Figure 7: Hysteresis loop of a 4-nm thick $\mathrm{Co}_{2} \mathrm{MnGe}$ film grown directly on sapphire a-plane at a substrate temperature of $300^{\circ} \mathrm{C}$ measured at $5 \mathrm{~K}$. 

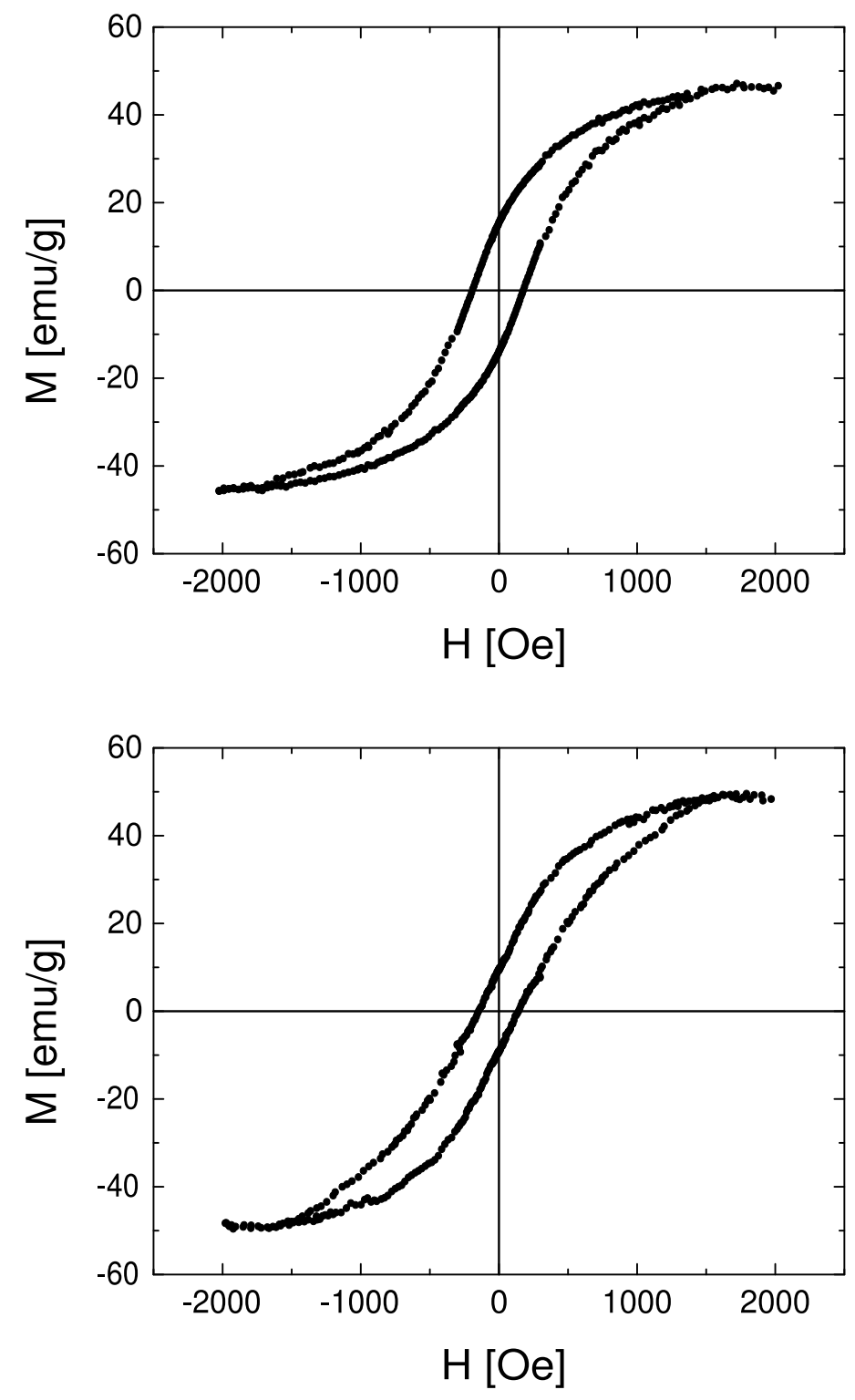

Figure 8: Hysteresis loops measured at $5 \mathrm{~K}$ of $\mathrm{V}(3 \mathrm{~nm}) / \mathrm{Co}_{2} \mathrm{MnGe}(4 \mathrm{~nm}) / \mathrm{V}(3 \mathrm{~nm})$ with the field applied parallel to the sample plane in the upper panel (a) and perpendicular to the sample plane in the bottom panel (b). 


\subsection{XMCD on $\mathrm{Co}_{2} \mathrm{MnGe}$}

In order to elucidate the microscopic origin of the moment reduction in the $\mathrm{Co}_{2} \mathrm{MnGe}$ Heusler alloy when prepared at low substrate temperatures (see figure 6) $\mathrm{x}$-ray magnetic circular dichroism (XMCD) is a very suitable experimental method as it allows an element specific study of the magnetism [74. We therefore prepared a $\mathrm{Co}_{2} \mathrm{MnGe}$ film specially designed for an XMCD study with 16-nm thickness grown on a V seed layer and with a 2-nm Au cap layer, all layers prepared at $T_{s}=300^{\circ} \mathrm{C}$. The saturation magnetization of the film as measured by SQUID magnetometry was found to be slightly smaller than the value given in Fig.5, it corresponds to a magnetic moment per formula unit of $2.3 \mu_{B}$ at room temperature and $2.98 \mu_{B}$ at $4 \mathrm{~K}$.

The XMCD measurements were performed at the bending magnet beamline PM3 at BESSY II (Berlin Germany) using the new ALICE chamber for spectroscopy and resonant scattering. 75]. The measurements were taken by the total electron yield (TEY) method. At an angle of incidence of $40^{\circ}$ saturation effects are small and the TEY is proportional to the absorption coefficient to a good approximation. During the experiment the helicity of the photons was fixed whereas the magnetization of the sample was switched by a magnetic field of $\pm 0.1 \mathrm{~T}$ thus providing the electron yield with the magnetization parallel $\left(\mathrm{Y}_{+}\right)$ and antiparallel $\left(\mathrm{Y}_{-}\right)$to the photon helicity. The $\mathrm{Y}_{+}$and $\mathrm{Y}_{-}$scans measured at the $L_{3}$ edge of $\mathrm{Mn}$ and Co are normalized to the flux of the incoming photon beam. The XMCD spectrum $\left(\mathrm{Y}_{+}-\mathrm{Y}_{-}\right)$at the $L_{2,3}$ edges of Mn and Co measured at room temperature is plotted in figure 9. The XMCD spectra contain quantitative information on the spin and orbital magnetic moments which can be extracted via the sum rule analysis [71. There are several sources of systematic errors in this analysis which might affect the absolute values for the magnetic moment questionable. This is first the number of holes in the $d$-band, which we can precisely take from electronic band structure calculations. Second, it is the neglecting of magnetic dipolar interactions in the model, which in our case seems justified because of the cubic symmetry of the $\mathrm{Co}_{2} \mathrm{MnGe}$ phase. Third, for the Mn atom there might be a mixing of the $L_{3}$ and the $L_{2}$ levels by the relatively strong $2 p-3 d$ Coulomb interactions. The correction factor $x$ taking this effect into consideration has been calculated ranging from $x=1$ for negligible $j j$-mixing to $x=1.5$ for strong $j j$-mixing [76]. Keeping these reservation in mind, the sum rule analysis yields for the case of the Co atom $m_{\text {spin }}=0.55 \mu_{B}$ for the spin magnetic moment and $m_{\text {orb }}=0.028 \mu_{B}$ for the orbital magnetic moment. For the case of the $\mathrm{Mn}$ atom the analysis yields $m_{\text {spin }}=0.98 \mu_{B}(1.47$ $\left.\mu_{B}\right)$ and $m_{\text {orb }}=0.056 \mu_{B}$, where for the first value it is assumed that $x=1.0$ holds, for the value given in brackets $x=1.5$ is assumed. Summing up all values for the atomic magnetic moments and extrapolating to $4 \mathrm{~K}$ we determine a saturation magnetic moment of $m=0.75 \mu_{B}$ for Co and $m=1.36 \mu_{B}\left(1.97 \mu_{B}\right)$ for $\mathrm{Mn}$ [71]. The moment for Co agrees reasonable with the theoretical value from band structure calculations, for the Mn atom the theoretical calculations give $m=3.6 \mu_{B}$ i.e. a much higher value than the experiment, irrespective of the exact value of the correction factor $x$. The magnetization data yielded a 


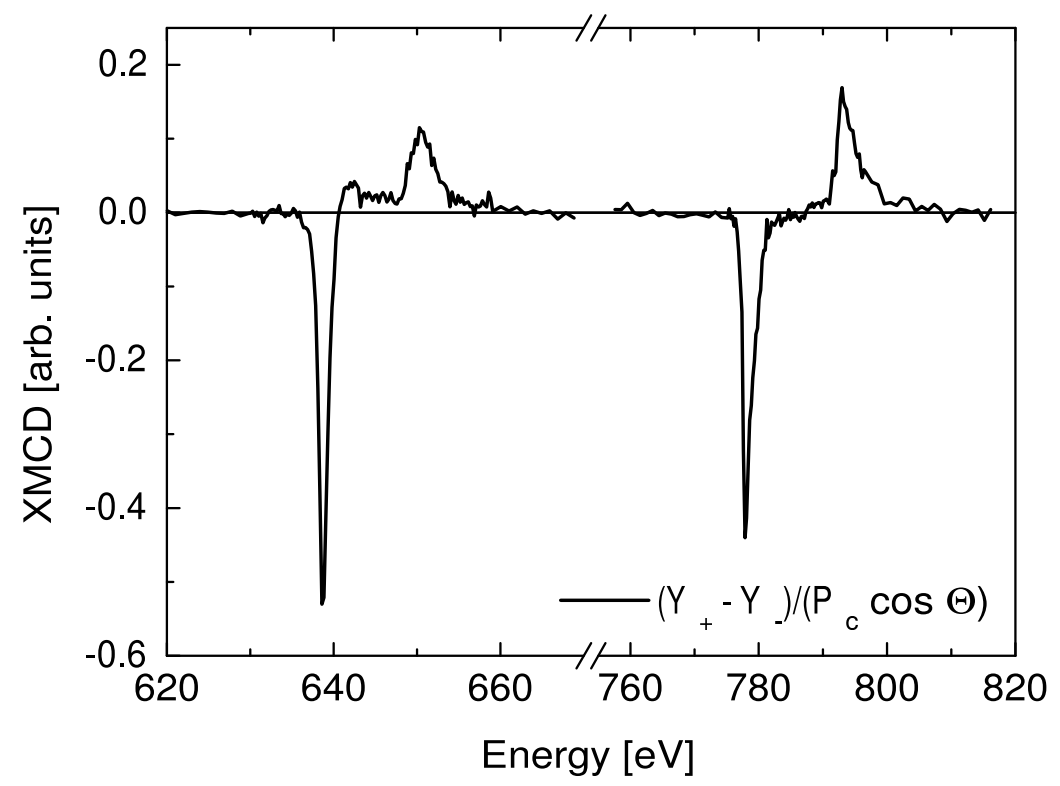

Figure 9: XMCD spectra of a 11-nm thick $\mathrm{Co}_{2} \mathrm{MnGe}$ film at the $\mathrm{Mn}_{3,2}$ and at the $\mathrm{Co} \mathrm{L}_{3,2}$ edge.

saturation magnetic moment of $2.98 \mu_{B}$ per $\mathrm{Co}_{2} \mathrm{MnGe}$ formula unit, the XMCD results yield $2.83 \mu_{B}\left(3.46 \mu_{B}\right)$, i.e. within the uncertainty range of the XMCD result the agreement is satisfactory.

As mentioned in the introduction, the theoretical model calculations 47, 39 show that antisite disorder in the $\mathrm{L} 2_{1}$ structure severely affects the $\mathrm{Mn}$ magnetic moments, since a Mn spin sitting on a regular Co position has a spin direction antiparallel to the other $\mathrm{Mn}$ and Co spins. This will strongly reduce the experimentally determined mean Mn moment. The value of the Co moment when sitting on a Mn position remains essentially unaffected. Thus the plausible hypothesis formulated above that a lower preparation temperatures causes site disorder in the $\mathrm{L} 2{ }_{1}$ structure and concomitantly a lowering of the saturation magnetization finds strong support from the XMCD results and the theoretical model calculations.

Summarizing this chapter, we have shown that with optimized preparation conditions high quality thick films of the $\mathrm{Co}_{2} \mathrm{MnGe}$ and the $\mathrm{Co}_{2} \mathrm{MnSn}$ phase can be grown. But if experimental constraints are imposed when preparing devices such as limits for the substrate temperature, non applicability of seed layers or if in devices very thin Heusler layers are needed, one faces problems. Site disorder in the interior of the films and mixing and disorder at interfaces have the tendency to lower the ferromagnetic magnetization. The magnetic behaviour of very small $\mathrm{Co}_{2} \mathrm{MnGe}$ grains as e.g. the nearly complete loss of ferromagnetism for $\mathrm{Co}_{2} \mathrm{MnGe}$ deposited on bare sapphire or the typical small 
particle magnetic behaviour in very thin $\mathrm{Co}_{2} \mathrm{MnGe}$ grown on $\mathrm{V}$ suggest that the grain boundaries are only weakly ferromagnetic or even non ferromagnetic.

\section{Multilayers $\left[\mathrm{Co}_{2} \mathrm{MnSn}(\mathrm{Ge}) / \mathrm{Au}(\mathrm{V})\right]_{n}$}

\subsection{Structural and magnetic properties}

Multilayers of the two Heusler phases $\mathrm{Co}_{2} \mathrm{MnGe}$ and $\mathrm{Co}_{2} \mathrm{MnSn}$ with $\mathrm{V}$ and $\mathrm{Au}$ as interlayers have been prepared by the same dual source rf-sputtering equipment described in section 2. For the multilayer preparation the substrate holder is swept between the two targets, if nothing else is stated, the number of bilayers prepared is $n=30$ starting with either $\mathrm{V}$ or $\mathrm{Au}$, and the films are protected by a $\mathrm{Al}_{2} \mathrm{O}_{3}$ cap layer. The substrate temperature was held fixed at $T_{S}=300^{\circ} \mathrm{C}$ for all multilayers, the deposition rates of the materials were the same as given in section 2 for the single layers. Although the structural quality of the Heusler layers improve at higher substrate temperatures, $T_{S}=300^{\circ} \mathrm{C}$ turned out to be the upper limit when strong interdiffusion at the interfaces must be avoided.

Using the natural gradient of the sputtering rate, the simultaneous preparation of up to 10 samples with the thickness of either the magnetic layer or the non magnetic layer altered is possible. The thickness can be varied by a factor of three and we exploit this feature e.g. for the preparation of several series of multilayers for the investigation of the thickness dependence of the magnetic interlayer coupling. In our previous investigations we have also tested the growth of Heusler multilayers with several other combinations of materials, including the Heusler compounds $\mathrm{Co}_{2} \mathrm{MnSi}$ and $\mathrm{Cu}_{2} \mathrm{MnAl}$ and $\mathrm{Cr}$ as interlayers [67, 65]. Here we concentrate on $\mathrm{Co}_{2} \mathrm{MnGe}$ and $\mathrm{Co}_{2} \mathrm{MnSn}$ with $\mathrm{Au}$ and $\mathrm{V}$ as interlayers which can be grown with best structural quality. The x-ray characterization of the multilayers was done by a standard thin film x-ray spectrometer or using synchrotron radiation at the Hasylab in Hamburg, Germany.

Figure [10 shows a low angle x-ray reflectivity scan measured on a $\left[\mathrm{Co}_{2} \mathrm{MnGe}(3 \mathrm{~nm}) / \mathrm{V}(2 \mathrm{~nm})\right]_{50}$ multilayer (the number in round brackets denotes the nominal thickness of the single layers, $n=50$ is the number bilayers) by synchrotron radiation at a wavelength of $0.177 \mathrm{~nm}$. One observes superlattice reflections up to the 4th order revealing a smooth layered structure. From a fit with the Parratt formalism shown by the displaces thin line one derives a thickness of $d_{V}=2.2 \mathrm{~nm}$ and $d_{\text {Heusler }}=2.8 \mathrm{~nm}$ and a roughness parameter of $\sigma=0.5 \mathrm{~nm}$ for $\mathrm{V}$ and $\sigma=0.7 \mathrm{~nm}$ for $\mathrm{Co}_{2} \mathrm{MnGe}$. Thus at the interfaces there is interdiffusion and/or topological roughness on a scale of about $0.6 \mathrm{~nm}$.

Figure $10 \mathrm{~b}$ depicts a scan across the $(220) /(110)$ Bragg reflection of the same multilayer. There is a rich structure with satellites up to $3 \mathrm{rd}$ order proving the growth of coherent $\mathrm{V}$ and $\mathrm{Co}_{2} \mathrm{MnGe}$ layers. The out of plane coherence length derived from the width of the Bragg peak is about $13 \mathrm{~nm}$ i.e. comprises about 3 bilayers. Figure 11 a shows the small angle reflectivity scan for a nominal $\left[\mathrm{Co}_{2} \mathrm{MnGe}(3 \mathrm{~nm}) / \mathrm{Au}(2.5 \mathrm{~nm})\right]_{50}$ multilayer. The fit with the Parratt formalism gives a thickness of $3.0 \mathrm{~nm}$ and $2.3 \mathrm{~nm}$ for the Heusler and Au layer respectively. 

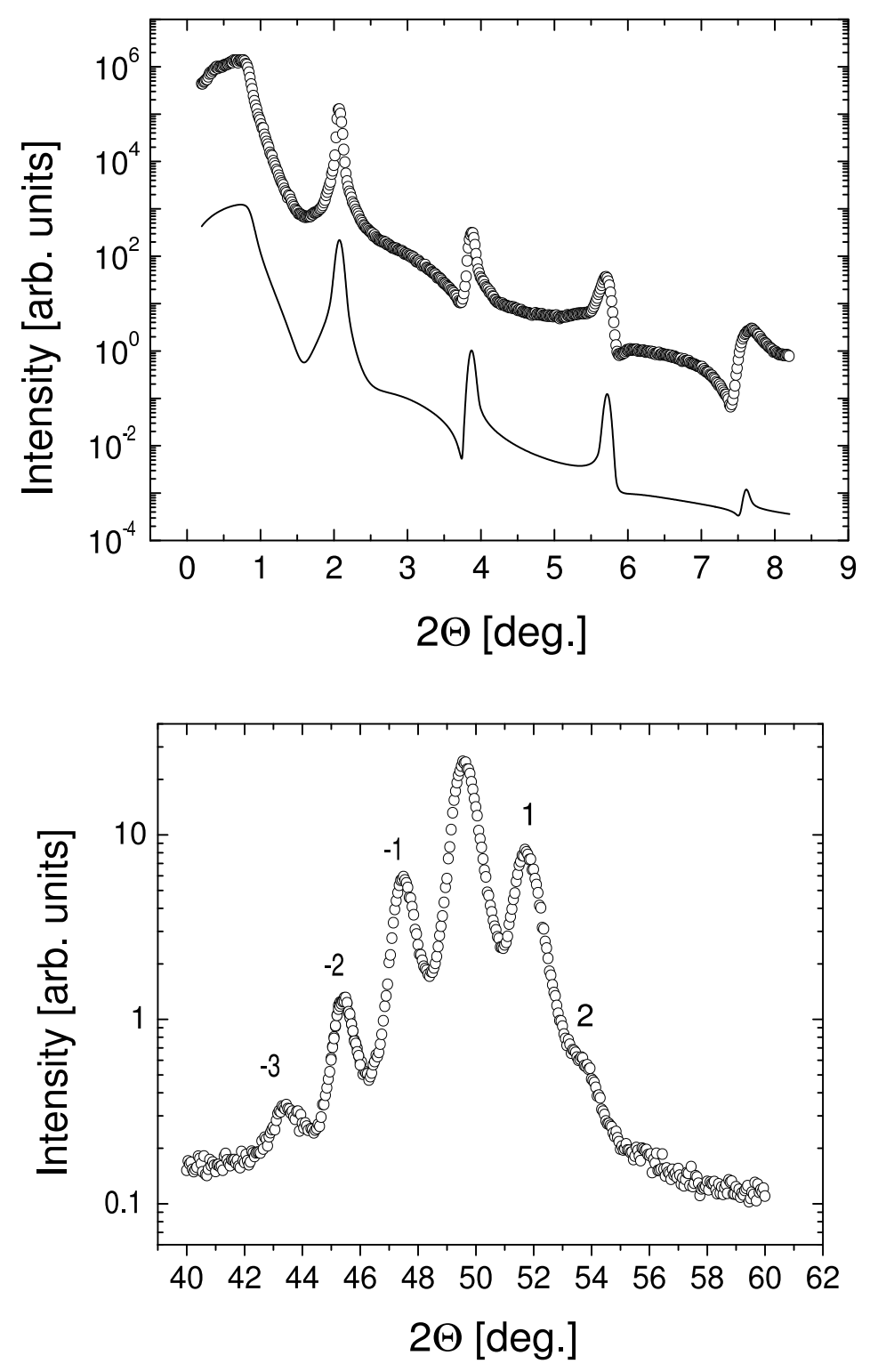

Figure 10: Upper panel (a): X-ray reflectivity scan of a $\left[\mathrm{Co}_{2} \mathrm{MnGe}(3 \mathrm{~nm}) / \mathrm{V}(2 \mathrm{~nm})\right]_{50}$ multilayer measured at a wavelength of the synchroton radiation $\lambda=1,77 \mathrm{~nm}$. The open symbols are the measured intensity, the straight line shows a simulation. Bottom panel: (b) Out of plane Bragg scan of the same multilayer at $\lambda=1,77 \mathrm{~nm}$. The numbers in the figure denote the order of the superlattice reflections and the order of the satellites. 

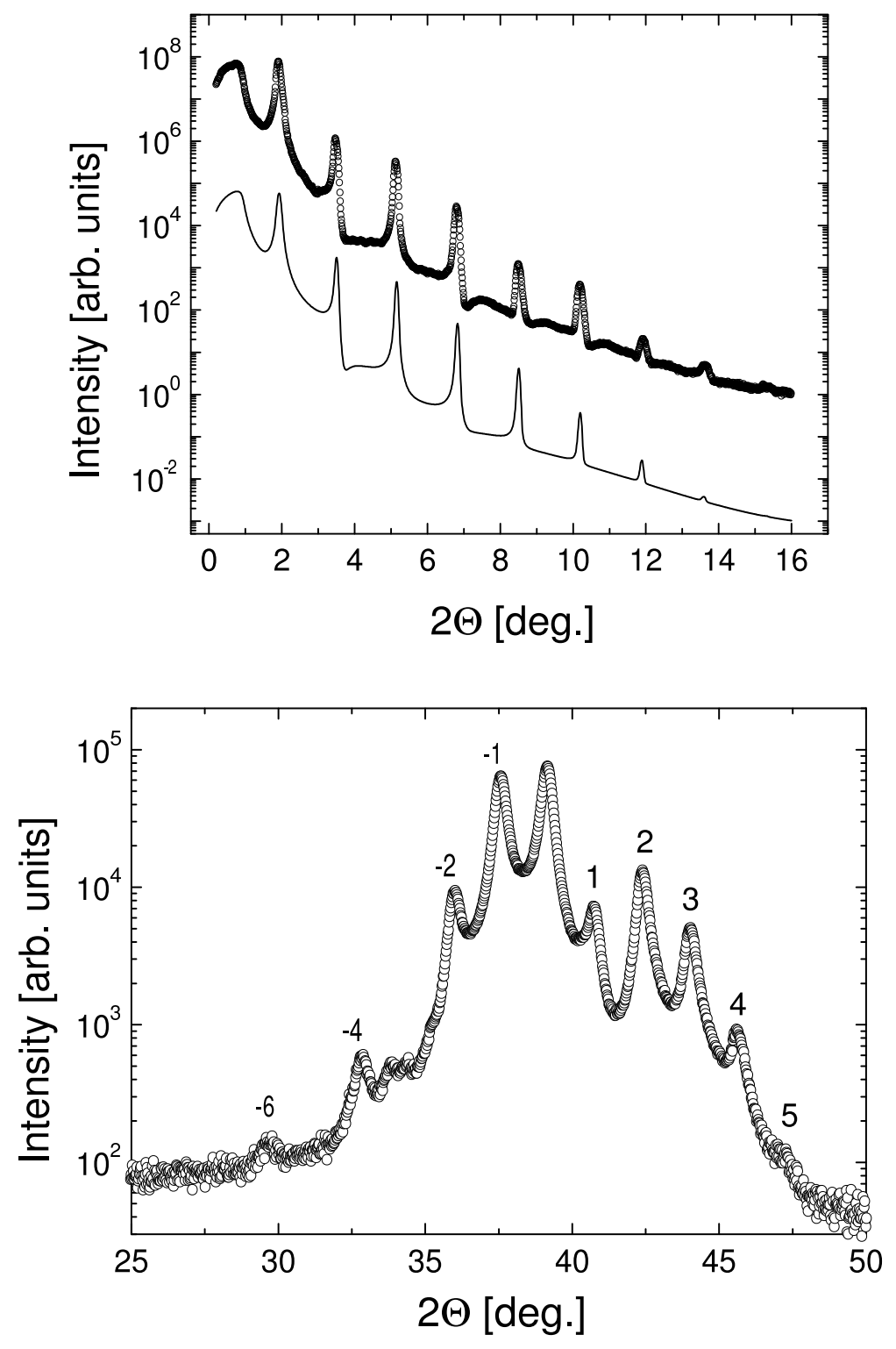

Figure 11: Upper panel (a): X-ray reflectivity scan of a $\left[\mathrm{Co}_{2} \mathrm{MnGe}(3 \mathrm{~nm}) / \mathrm{Au}(2.5 \mathrm{~nm})\right]_{50}$ multilayer measured at $\lambda=1,54 \mathrm{~nm}$. The open symbols are the measured intensity, the straight line shows a simulation. Bottom panel (b): Out of plane Bragg scan of the same multilayer at $\lambda=1,54 \mathrm{~nm}$. 
As already evident from the narrower superlattice reflection peaks, the quality of the layered structure is even better than for the $\left[\mathrm{Co}_{2} \mathrm{MnGe} / \mathrm{V}\right]$ multilayer of figure 10 a. This is corroborated by the simulation which gives a roughness parameter $\sigma_{A u}=0.5 \mathrm{~nm}$ and $\sigma_{\text {Heusler }}=0.3 \mathrm{~nm}$. The Bragg scan (figure11) at the $(111) /(220)$ Bragg position also reveals an out of plane coherent crystalline lattice with a coherence length of $14 \mathrm{~nm}$.

We have also grown successfully $\left[\mathrm{Co}_{2} \mathrm{MnSn} / \mathrm{V}\right]$ and $\left[\mathrm{Co}_{2} \mathrm{MnSn} / \mathrm{Au}\right]$ multilayers with comparable quality [66] i.e. with an out of plane coherence length and a roughness parameter similar to the $\left[\mathrm{Co}_{2} \mathrm{MnGe} / \mathrm{V}\right]$ and $\left[\mathrm{Co}_{2} \mathrm{MnGe} / \mathrm{Au}\right]$ multilayers. Multilayers of similar quality can be grown in the thickness range of down to $1 \mathrm{~nm}$ for the Heusler compounds and the interlayers, for a smaller thickness the layered structure and the crystalline coherence is gradually lost.

The magnetic properties of the multilayers were studied by measurements of the magnetic hysteresis loops. Figures 12 and 13 show examples of hysteresis loops from the $\left[\mathrm{Co}_{2} \mathrm{MnGe} / \mathrm{Au}\right]$, the $\left[\mathrm{Co}_{2} \mathrm{MnSn} / \mathrm{Au}\right]$, the $\left[\mathrm{Co}_{2} \mathrm{MnGe} / \mathrm{V}\right]$ and the $\left[\mathrm{Co}_{2} \mathrm{MnSn} / \mathrm{V}\right]$ multilayer systems at different temperatures. Several important features should be noted.

The saturation magnetization measured at $5 \mathrm{~K}$ is smaller than the value for the bulk compound, the reduction is comparable to that we observed in single films of the same systems in section 2, The coercive field is strongly increasing at low temperatures from values of typically $H_{c}=50$ Oe at $60 \mathrm{~K}$ to several hundred Oe at $4 \mathrm{~K}$. This effect is shown in more detail in figure 14 where the coercive force drastically increases below $50 \mathrm{~K}$. This feature is often observed in magnetically inhomogeneous films and indicates that thermal activation plays an important role in the remagnetization processes at higher temperatures. Interestingly for the multilayers with $\mathrm{V}$ in figure 13 there is no observable magnetic remanence at higher temperature, the magnetization curve is completely reversible for temperatures above about $150 \mathrm{~K}$. On the other hand for the multilayers with Au (figure 12) there is a hysteresis with a finite remanent magnetization up to the ferromagnetic Curie temperature, as it should be for a normal ferromagnetic compound. Most of our multilayers possess a growth induced uniaxial magnetic anisotropy similar to the thick films discussed in section 2 2 , but with a definitely smaller amplitude of the order of 20 Oe for the anisotropy fields. For the hysteresis curve measurements in figure 12 the field is applied along the magnetic easy axis, thus the vanishing remanent magnetization cannot simply be explained by a magnetic anisotropy field perpendicular to the field direction.

We prepared series of 10 multilayer samples with either the thickness of the Heusler compound or the thickness of the $\mathrm{V}$ or Au layer varied between $1 \mathrm{~nm}$ and $3 \mathrm{~nm}$ and systematically studied the hysteresis curves. These results are summarized in the next three figures. In figure 15 we have plotted the relative remanent magnetization for $\left[\mathrm{Co}_{2} \mathrm{MnGe} / \mathrm{V}\right]$ and $\left[\mathrm{Co}_{2} \mathrm{MnSn} / \mathrm{Au}\right]$ multilayers determined at a temperature of $200 \mathrm{~K}$ as a function of the thickness of the non magnetic interlayers. We find no systematic variation with the thickness, for the multilayers with $\mathrm{Au}$ the remanent magnetization is about $60 \%$ of the saturation magnetization independent of the thickness of the Au layer, for $\left[\mathrm{Co}_{2} \mathrm{MnGe} / \mathrm{V}\right]_{n}$ 

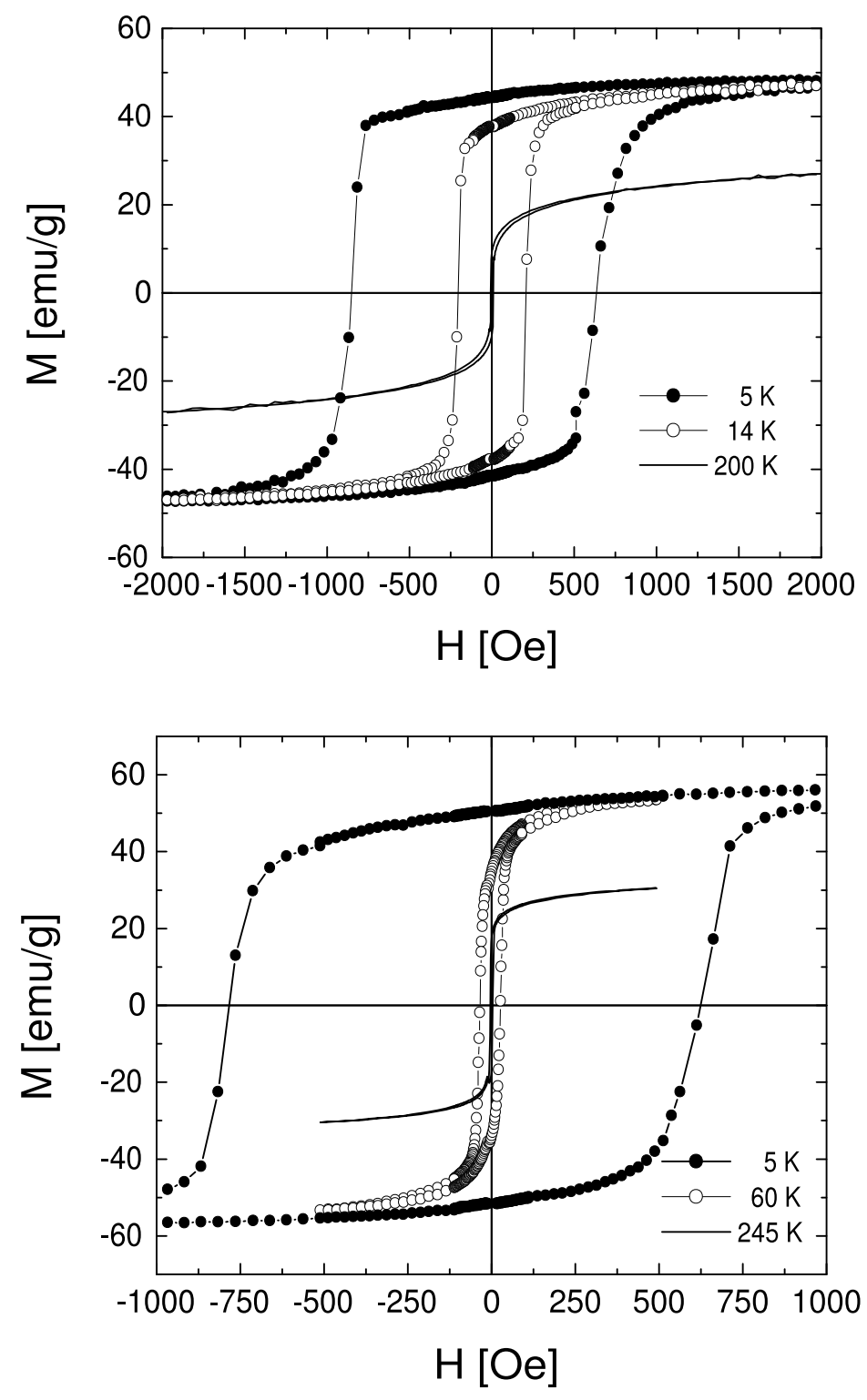

Figure 12: Hysteresis loops of the multilayers $\left[\mathrm{Co}_{2} \mathrm{MnGe}(2.2 \mathrm{~nm}) / \mathrm{Au}(3 \mathrm{~nm})\right]_{30}$ in the upper panel (a) and $\left[\mathrm{Co}_{2} \mathrm{MnSn}(3 \mathrm{~nm}) / \mathrm{Au}(1.5 \mathrm{~nm})\right]_{30}$ in the bottom panel (b) measured at different temperatures given in the figure. 

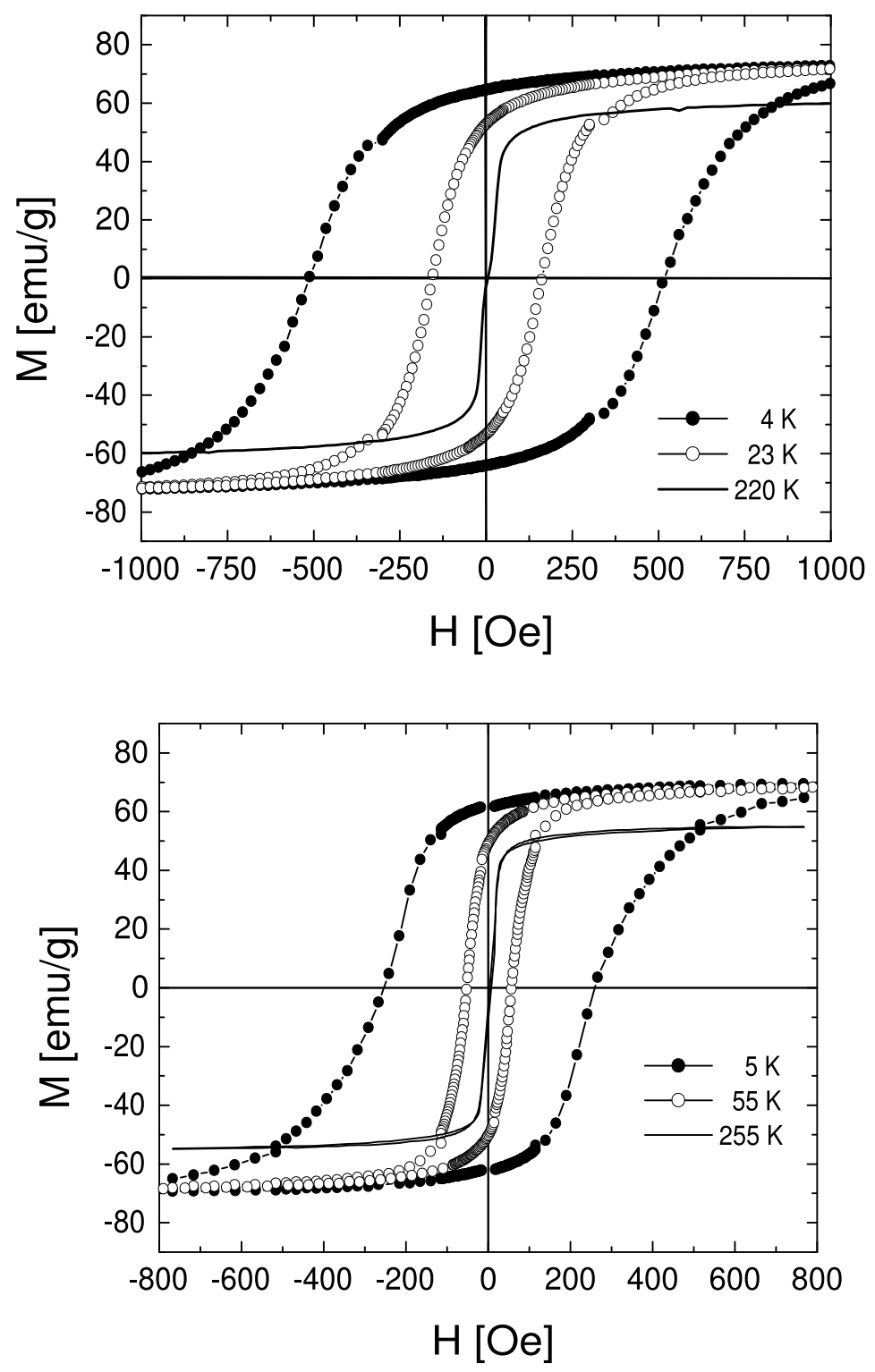

Figure 13: Hysteresis loops measured at different temperatures for the samples $\left[\mathrm{Co}_{2} \mathrm{MnGe}(3 \mathrm{~nm}) / \mathrm{V}(3 \mathrm{~nm})\right]_{30}$ in the upper panel (a) and $\left[\mathrm{Co}_{2} \mathrm{MnSn}(3 \mathrm{~nm}) / \mathrm{V}(3 \mathrm{~nm})\right]_{30}$ in the bottom panel (b). 


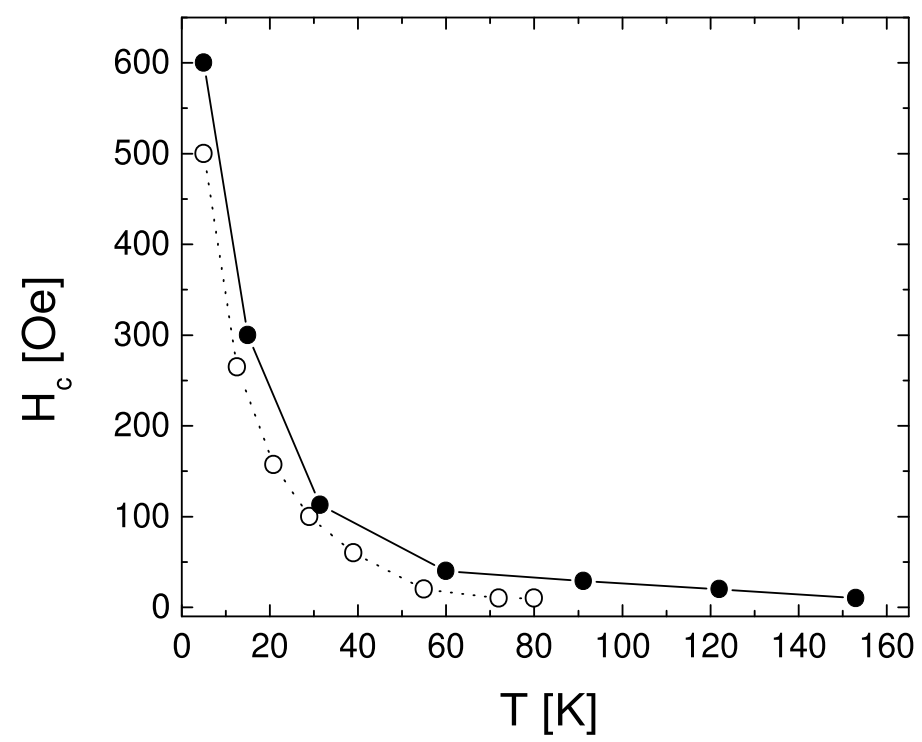

Figure 14: Coercive field versus temperature for the multilayers $\left[\mathrm{Co}_{2} \mathrm{MnSn}(3 \mathrm{~nm}) / \mathrm{Au}(1.5 \mathrm{~nm})\right]_{30}$ (solid circles) and $\left[\mathrm{Co}_{2} \mathrm{MnGe}(3 \mathrm{~nm}) / \mathrm{V}(4 \mathrm{~nm})\right]_{50}$ (open circles). 


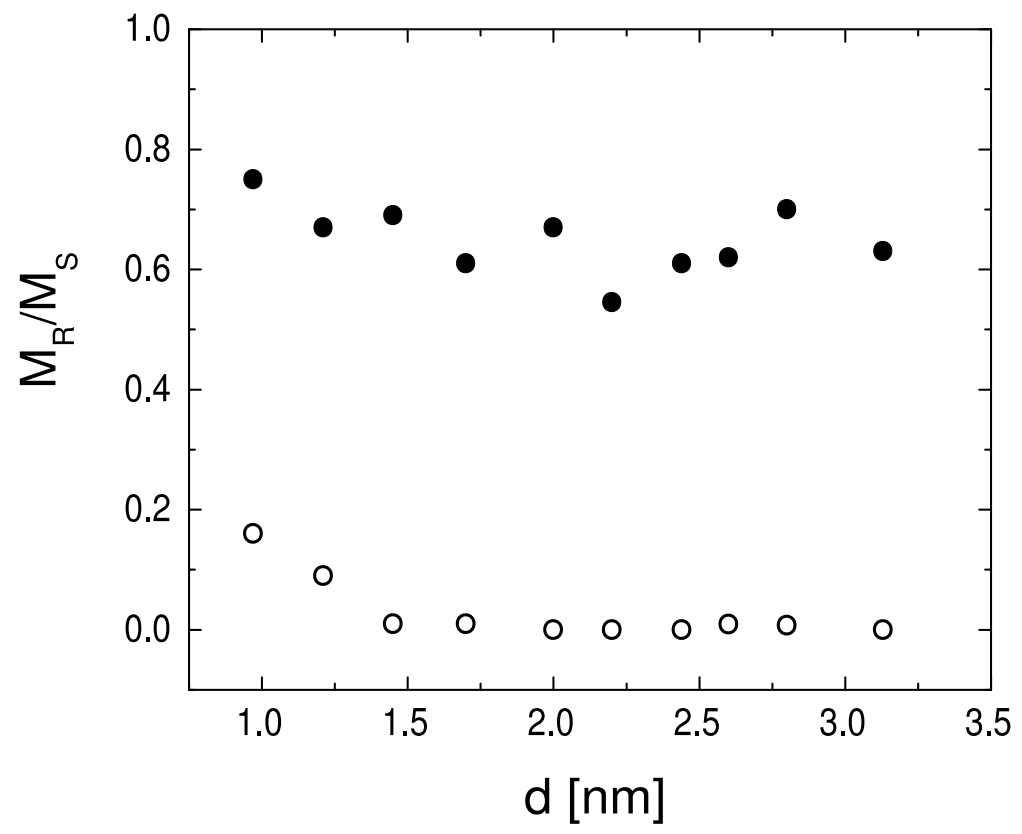

Figure 15: Relative remanent magnetization for $\left[\mathrm{Co}_{2} \mathrm{MnSn}(3 \mathrm{~nm}) / \mathrm{Au}(d)\right]_{30}$ (solid circles) and $\left[\mathrm{Co}_{2} \mathrm{MnGe}(3 \mathrm{~nm}) / \mathrm{V}(d)\right]_{30}$ (open circles) multilayers determined at $T=200 \mathrm{~K}$ versus thickness of the non magnetic interlayer $d$. 


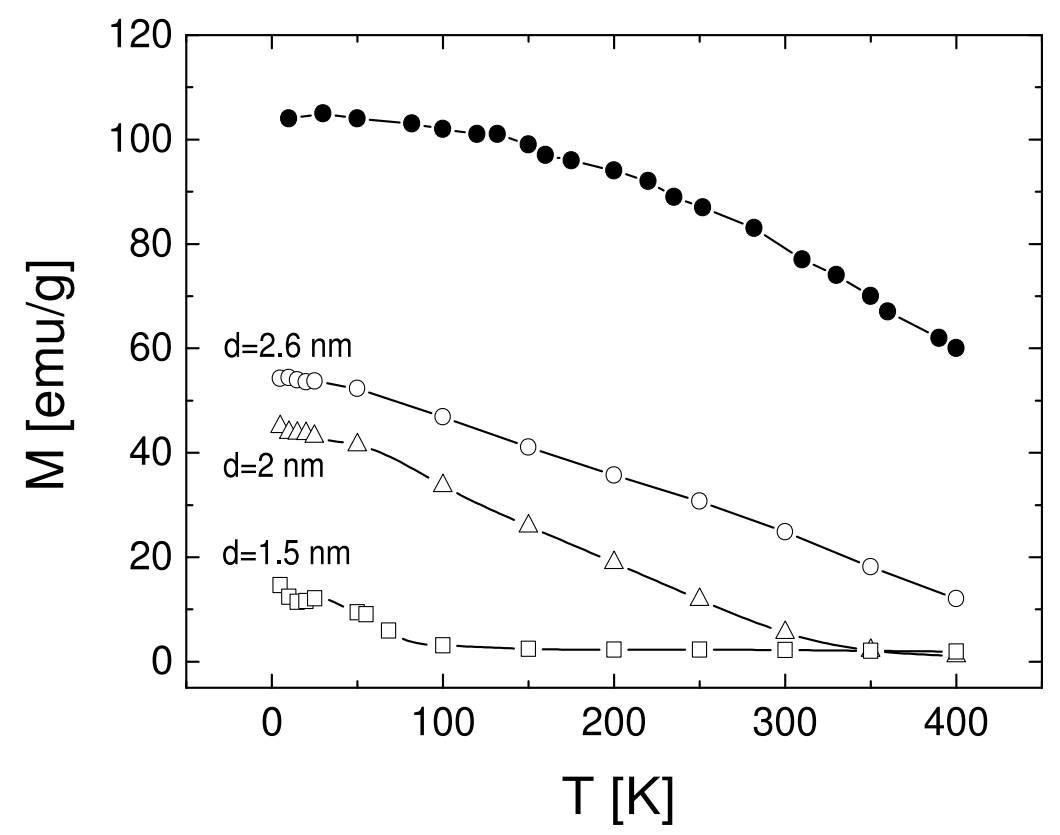

Figure 16: Ferromagnetic saturation magnetization of bulk $\mathrm{Co}_{2} \mathrm{MnGe}$ (solid circles) and $\left[\mathrm{Co}_{2} \mathrm{MnGe}(d) / \mathrm{Au}(3 \mathrm{~nm})\right]_{30}$ multilayers (open symbols) with varied $\mathrm{Co}_{2} \mathrm{MnGe}$ layer thickness $d$ (given in the figure) versus temperature.

the remanent magnetization vanishes for all thicknesses above $d=1.5 \mathrm{~nm}$. Thus there is no indication of an oscillation of the interlayer exchange interaction in $\left[\mathrm{Co}_{2} \mathrm{MnSn} / \mathrm{Au}\right]_{n}$ which in the case of an antiferomagnetic coupling should give a lowering of the remanent magnetization. Similarly in $\left[\mathrm{Co}_{2} \mathrm{MnGe} / \mathrm{V}\right]_{n}$ the vanishing remanent magnetization could indicate an af coupling, but then the af coupling would be independent of the interlayer thickness, which does not at all fit into the scheme of an IEC mechanism [72]. We will come back to this question in section 3.3 below. In figure 16 we have plotted the ferromagnetic saturation magnetization measured in a field of $2 \mathrm{kOe}$ when varying the thickness of the $\mathrm{Co}_{2} \mathrm{MnGe}$ layers while keeping the thickness of the Au layers constant. It is apparent that the saturation magnetization and the ferromagnetic Curie temperatures are much lower than for the bulk $\mathrm{Co}_{2} \mathrm{MnGe}$ phase and continuously decrease further with decreasing film thickness. For a layer thickness of $\mathrm{Co}_{2} \mathrm{MnGe}$ of $d=2.6 \mathrm{~nm}$ we estimate a ferromagnetic Curie temperature $T_{c}$ of about $500 \mathrm{~K}$, for $d=2 \mathrm{~nm}$ we get $T_{c} \sim 340 \mathrm{~K}$ and for $d=1.5 \mathrm{~nm} T_{c} \sim 90 \mathrm{~K}$. For the latter sample the saturation magnetization is very low and the magnetic ground state is a spin glass state rather than a ferromagnetic state (see next section). Finally in figure 17 we have plotted the ferromagnetic saturation mag- 


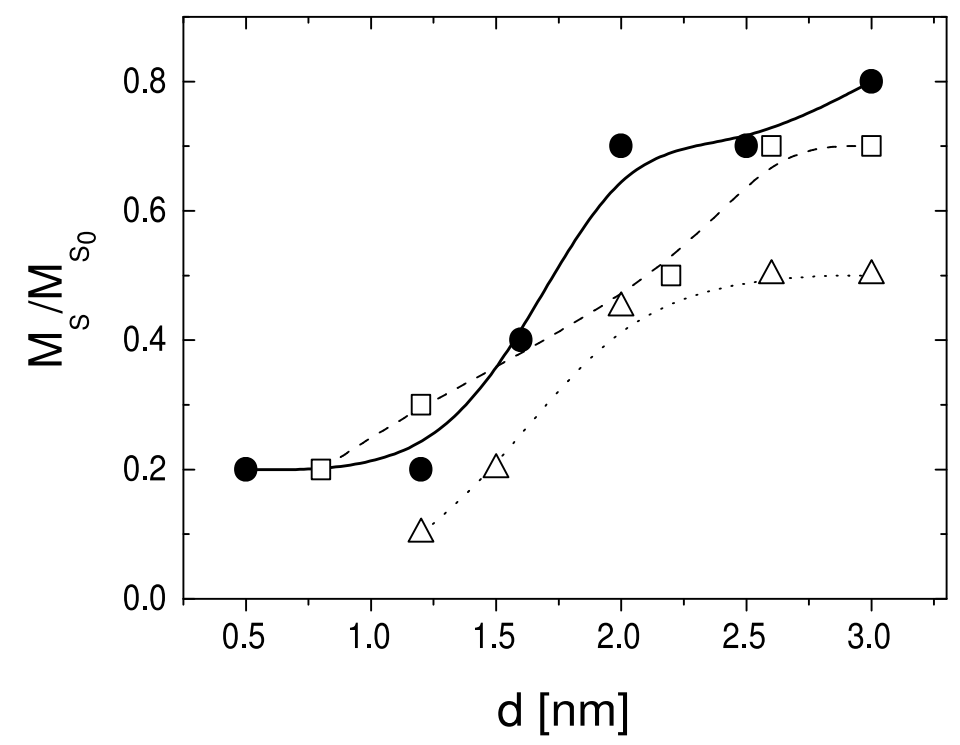

Figure 17: Relative saturation magnetization versus the thickness of the $\mathrm{Co}_{2} \mathrm{MnGe}$ and $\mathrm{Co}_{2} \mathrm{MnSn}$ layer for multilayers $\left[\mathrm{Co}_{2} \mathrm{MnSn}(d) / \mathrm{V}(3 \mathrm{~nm})\right]_{30}$ (circles), $\left[\mathrm{Co}_{2} \mathrm{MnGe}(d) / \mathrm{V}(3 \mathrm{~nm})\right]_{30}$ (squares) and $\left[\mathrm{Co}_{2} \mathrm{MnSn}(d) / \mathrm{Au}(3 \mathrm{~nm})\right]_{30}$ (triangles).

netization measured at $T=5 \mathrm{~K}$ and $H=2 \mathrm{kOe}$ as a function of the magnetic layer thickness for the different multilayer systems under study here. One finds that for a layer thickness of $d=3 \mathrm{~nm}$ the saturation magnetization reaches only 50 to $80 \%$ of the bulk saturation magnetization, depending on the combination. Typically below $d=1.5 \mathrm{~nm}$ the ferromagnetic moment breaks down completely, suggesting that at this thickness mixing and disorder from both sides of the ferromagnetic layer destroys the ferromagnetic ground state completely.

Summarizing this section, we have shown that high quality multilayers of the Heusler compounds $\mathrm{Co}_{2} \mathrm{MnGe}$ and $\mathrm{Co}_{2} \mathrm{MnSn}$ can be grown on sapphire aplane. The ferromagnetic saturation magnetization is definitely lower than the bulk value but similar to what we have observed for the very thin single films. The reduction of the magnetization has two different sources. First there are many antisite defects since the multilayers are prepared at $300^{\circ} \mathrm{C}$ and not at the much higher optimum temperature. Second, there is an additional drastic breakdown of the saturation magnetization at the interfaces due to strong disorder and intermixing here. From the investigations of the multilayers with very thin magnetic films we may conclude that typically $0.7 \mathrm{~nm}$ of the Heusler layers at the interfaces contribute only a small magnetization and are not ferromagnetic. This is supported by the results of the small angle x-ray reflectivity, from which we derived an interface roughness of the same order of magnitude. When varying the thickness of the non magnetic interlayers $\mathrm{Au}$ and $\mathrm{V}$ we find no 
indication of an oscillating interlayer exchange coupling, but for the case of the $\mathrm{V}$ interlayers there is an interesting anomalous behaviour, namely a vanishing remanent magnetization over a broad thickness range which will be the subject of the section 3.3 .

\subsection{Exchange bias in $\left[\mathrm{Co}_{2} \mathrm{MnGe} / \mathrm{Au}\right]$ multilayers}

Apart from the more general magnetic properties of the Co-based Heusler multilayers presented in the previous section, there are intriguing peculiarities in the magnetic order of these multilayers which we want to discuss in this and the next section.

After cooling in a magnetic field most of the Co-based Heusler multilayers which we have studied exhibit asymmetric magnetic hysteresis loops shifted along the magnetic field axis, evidencing the existence of an unidirectional exchange anisotropy, nowadays dubbed "exchange bias" [77. This is an effect known to occur at interfaces between an antiferromagnet (af) and a ferromagnet (f), the classical example being the $\mathrm{Co} / \mathrm{CoO}$ interface [77]. The exchange bias phenomenon attracted considerable new interest in recent years and there is a much deeper understanding of it now than it was ten years before, although a quantitative theoretical model is still lacking. The exchange bias sets in at a blocking temperature $T_{B}$ slightly below the Néel temperature $T_{N}$ of the antiferromagnet and there is general agreement in the literature that the spins at the $\mathrm{f} /$ af interface which are coupled to both, the ferromagnet and the antiferromagnet, cause the exchange bias effect. The coupling to the ferromagnet in saturation defines the direction of the exchange bias field $H_{E B}$. Upon cooling through $T_{B}$ the interface spins are blocked and keep the direction of $H_{E B}$ fixed. Theoretical models developed for the exchange bias effect in recent years focus on different aspects of the complex problem. The domain state model [78] assumes that the domain formation in the antiferromagnet is the most essential point, other models start from the frustrated, weakly coupled spins at the interface or at domain walls of the antiferromagnet [80, 79].

We have observed an exchange bias shift of the hysteresis loop in multilayers of $\mathrm{Co}_{2} \mathrm{MnSn}$ and $\mathrm{Co}_{2} \mathrm{MnGe}$ when combined with $\mathrm{Au}, \mathrm{Cr}$ or $\mathrm{Cu}_{2} \mathrm{MnAl}$ [65]. Only with $\mathrm{V}$ interlayers it does not occur. Since certainly there is no antiferromagnetism in these multilayers, the existence of $H_{E B}$ is puzzling at the first glance.

Before coming back to this question we shortly introduce the main experimental results for the example of the $\left[\mathrm{Co}_{2} \mathrm{MnGe} / \mathrm{Au}\right]$ multilayer system. In figure 18 we show the hysteresis loop measured at $2 \mathrm{~K}$ after field cooling in an applied field of $2 \mathrm{kOe}$. The loop is found to be shifted by a rather large exchange bias field $H_{E B}=500$ Oe. Similar to many other exchange bias systems [77] there is a strong relaxation of $H_{E B}$ when sweeping the magnetic field several times (see sweep nb. 1 to 4 in figure 18). The exchange bias effect sets in rather sharply at a blocking temperature of $15 \mathrm{~K}$ (figure 19), showing that the magnetic exchange interactions defining $T_{B}$ are rather weak. We have also tested the dependence of $H_{E B}$ on the thickness of the magnetic layer $d_{m}$ and 


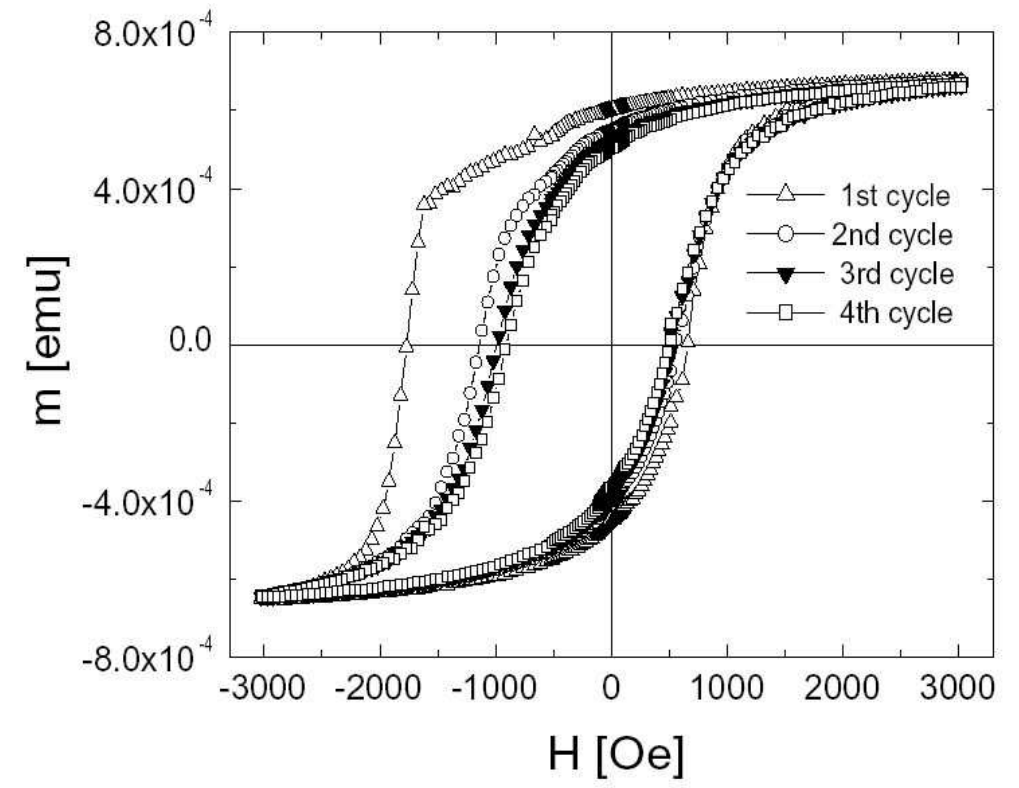

Figure 18: Sequence of magnetic hysteresis loops of the multilayer $\left[\mathrm{Co}_{2} \mathrm{MnGe}(2.6 \mathrm{~nm}) / \mathrm{Au}(3 \mathrm{~nm})\right]_{30}$ measured at $2 \mathrm{~K}$ after field cooling in $H=$ $2 \mathrm{kOe}$.

found $H_{E B} \sim 1 / d_{m}$ as usual for exchange bias systems and proving that the exchange anisotropy originates from interface spins.

There is one enlightening experiment which is difficult to realize in conventional $\mathrm{f} / \mathrm{af}$ exchange bias systems and which we show in figure 20. When sweeping the external field to $-4 \mathrm{~T}$ at $5 \mathrm{~K}$, one can nearly eliminate $H_{E B}$. Simultaneously the excess magnetization (asymmetry of the loop along the magnetization axis) vanishes, directly proving the intimate correlation of the excess positive magnetization and $H_{E B}$. It seems quite natural to conclude that interface spins determining $H_{E B}$ are rotated out of their metastable orientation when sweeping the field up to $4 \mathrm{~T}$. Now coming back to the question concerning the origin of the exchange bias shift in our multilayers, the mere existence of $H_{E B}$ shows that at the $\mathrm{Co}_{2} \mathrm{MnGe} / \mathrm{Au}$ interface antiferromagnetic exchange interactions must be present. The excess positive magnetization which vanishes after magnetizing to high negative fields shows that there are spins at the interface with a component antiparallel to the ferromagnet. Adopting the point of view of localized models for magnetic exchange interactions, there seems to be an antiferromagnetic superexchange type of interaction at the interface e.g. an af $\mathrm{Co}-\mathrm{Au}-\mathrm{Co}$ superexchange or an af $\mathrm{Co}-\mathrm{Au}-\mathrm{Mn}$ superexchange. This interaction competing with the ferromagnetic exchange of the pure $\mathrm{Co}_{2} \mathrm{MnGe}$ phase creates a few monolayers with frustrated spins and spin glass type of order at the interface. Actually the magnetic order of very thin $\mathrm{Co}_{2} \mathrm{MnGe}$ layers with a thickness 


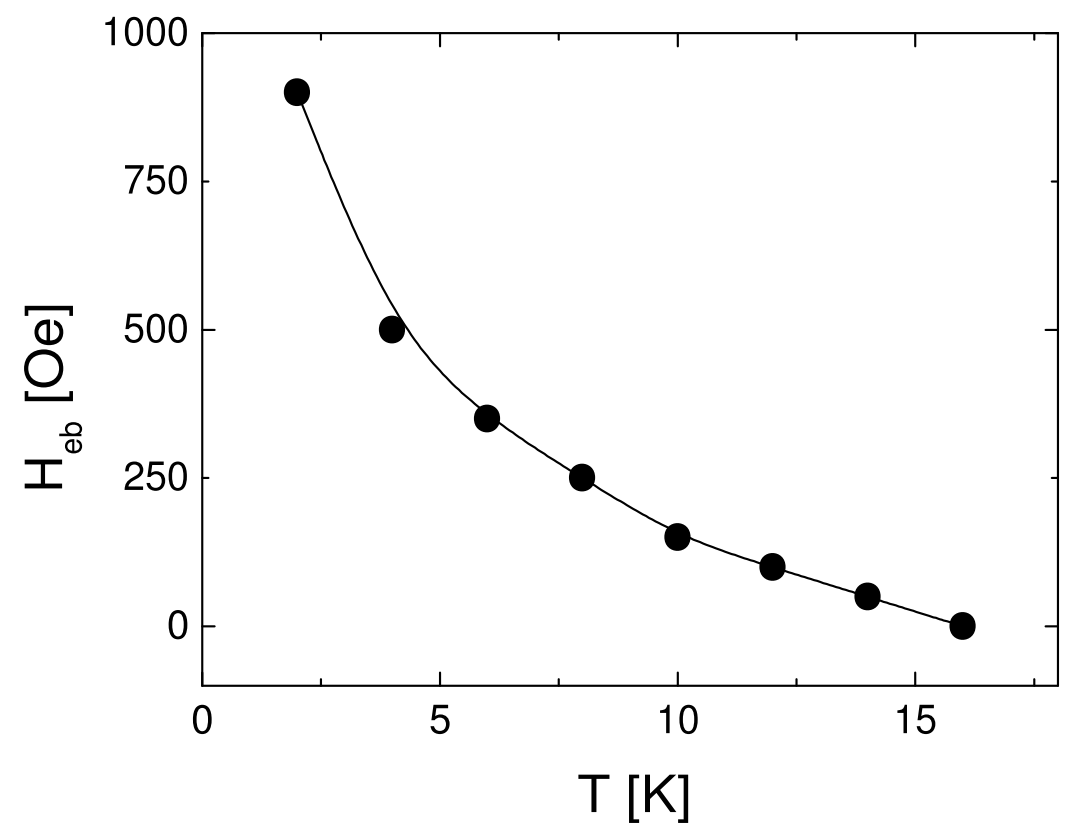

Figure 19: Exchange bias field versus temperature for the multilayer $\left[\mathrm{Co}_{2} \mathrm{MnGe}(2.6 \mathrm{~nm}) / \mathrm{Au}(3 \mathrm{~nm})\right]_{30}$ for a cooling field $H=200$ Oe.

of the order of $1 \mathrm{~nm}$ exhibit all ingredients of spin glass order (figure 21). This spin glass phase takes over the role of the antiferromagnet in conventional $\mathrm{f} / \mathrm{af}$ exchange bias systems. The spin glass provides a source of weakly coupled spins which are blocked at the spin glass freezing temperature $T_{f}$, which replaces the blocking temperature $T_{B}$ of the $\mathrm{f} /$ af interface.

The experimental fact that the exchange bias effect occurs in many Co based Heusler multilayers shows that the existence of antiferromagnetic interactions and spin glass order are the rule rather than the exception in these multilayers.

\subsection{Antiferromagnetic interlayer coupling in $\left[\mathrm{Co}_{2} \mathrm{MnGe} / \mathrm{V}\right]$ multilayers}

It is an interesting principal question whether in the Co Heusler based multilayers an oscillatory interlayer exchange coupling (IEC) of the type existing in multilayers combined of the $3 d$ transition element ferromagnets and normal metals [72] can be observed, too. Thus we carefully inspected the magnetic hysteresis loops of the Co-based Heusler multilayers from section 3.1 in order to find indications for an IEC mechanism. We were successful in the $\left[\mathrm{Co}_{2} \mathrm{MnGe} / \mathrm{V}\right] \mathrm{mul}-$ tilayer system, where a vanishing remanence at room temperature (see fiogure 


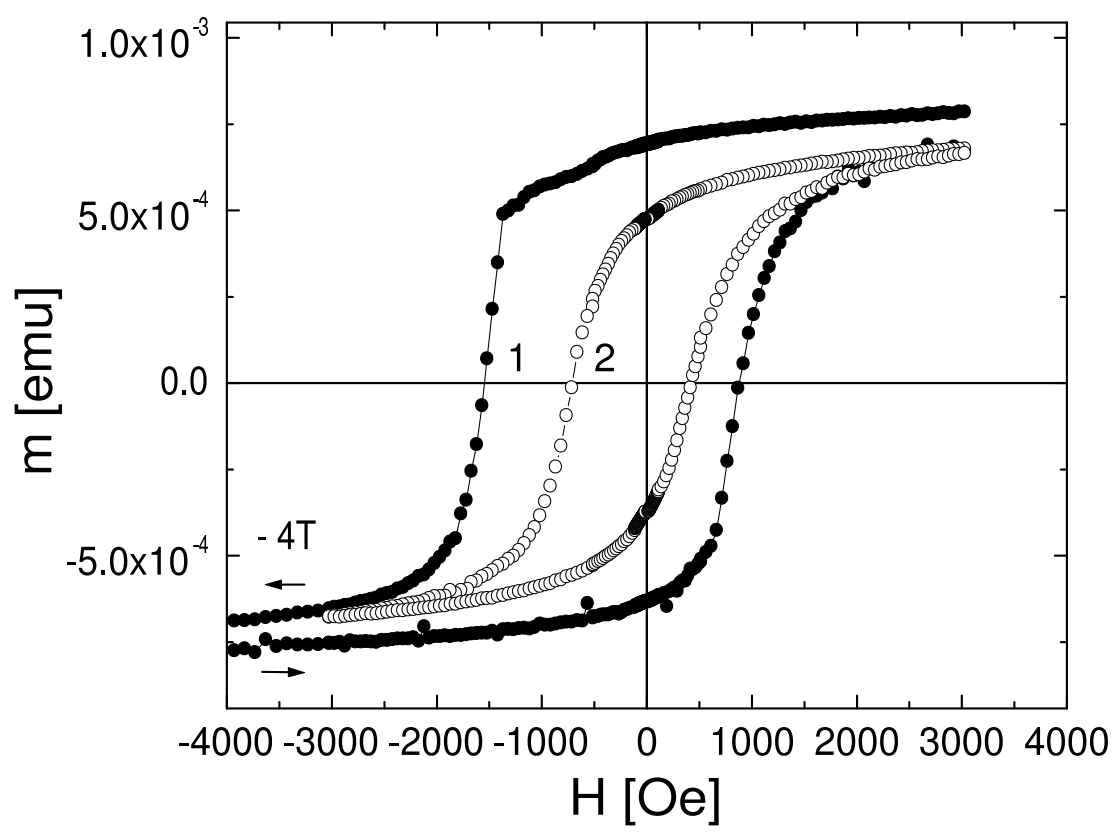

Figure 20: Magnetic hysteresis loops of the multiayer $\left[\mathrm{Co}_{2} \mathrm{MnGe}(2.6 \mathrm{~nm}) / \mathrm{Au}(3 \mathrm{~nm})\right]_{30}$ measured at $2 \mathrm{~K}$ after field cooling in $H=40 \mathrm{kOe}$. The labels in the figure denote the number of the field cycles. The field has been driven to $-40 \mathrm{kOe}$ during the first field cycle.

[13a) is seen, giving a first indication of an antiferromagnetic IEC.

We then started a systematic investigation of the magnetic order in this system by magnetic neutron reflectometry. The experiments were carried out at the ADAM reflectometer at the ILL in Grenoble. This instrument is equipped with neutron spin analysers so that spin polarized neutron reflectivity (PNR) measurements can be performed. PNR gives additional and important information about the magnetization direction in the film [81, 82, 83], since a magnetization direction parallel (or antiparallel) to the neutron spin polarization causes scattering with the polarization direction unchanged (up-up $(+,+)$ and down-down $(-,-)$ channels) only, whereas a magnetization component perpendicular to the spin quantization axis causes spin flip scattering appearing in the down-up $(-,+)$ and up-down $(+,-)$ channels.

Figure 22 displays (unpolarized) neutron reflectivity scans for a series of $\left[\mathrm{Co}_{2} \mathrm{MnGe} / \mathrm{V}\right]$ multilayers with different thickness of the $\mathrm{V}$ layer $d_{V}$ ranging from $1.5 \mathrm{~nm}$ to $4 \mathrm{~nm}$. The observation of the half order peak clearly proves the existence of an antiferromagnetic (af) interlayer coupling. The af coupling is observed for all multilayers with an interlayer thickness $\mathrm{d}_{V}$ smaller than 3 


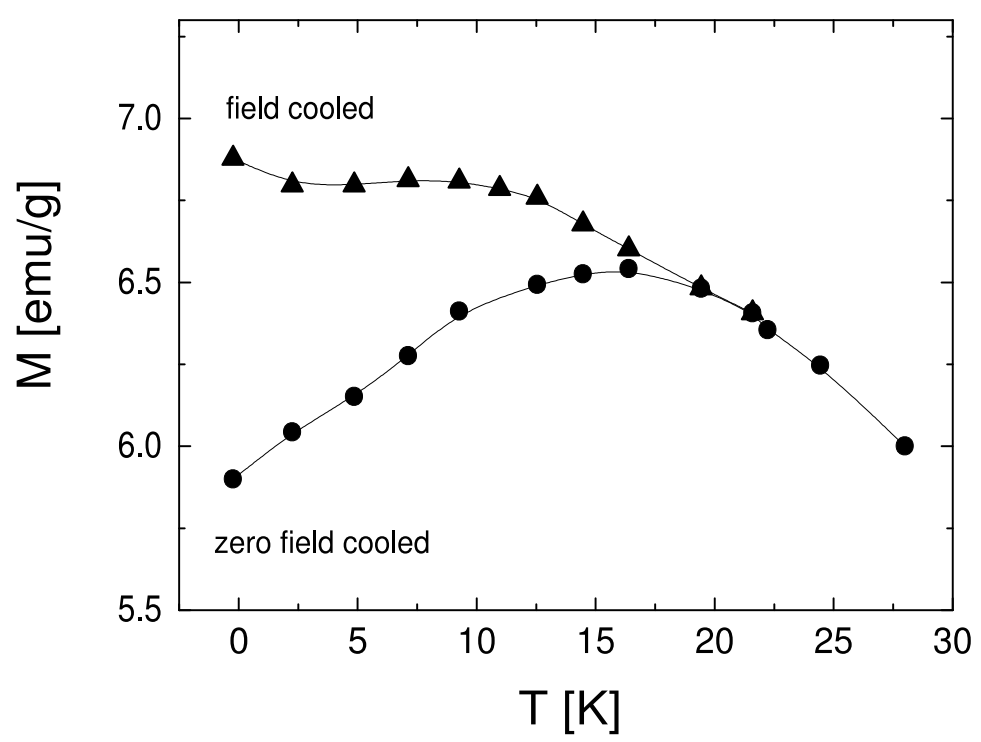

Figure 21: Field cooled and zero field cooled magnetization for the sample $\left[\mathrm{Co}_{2} \mathrm{MnGe}(1.2 \mathrm{~nm}) / \mathrm{Au}(3 \mathrm{~nm})\right]_{30}$ in a magnetic field of 500 Oe.

$\mathrm{nm}$, we find no indication of an oscillatory IEC in this thickness range. We also studied other, intermediate $\mathrm{V}$ thicknesses by magnetization measurements and found that the coupling is always antiferromagnetic. At this point serious doubts arise whether actually the IEC mechanism is responsible for the af interlayer coupling, since the oscillatory character of this coupling is an intrinsic property of the geometry of the Fermi surface of the interlayers [72].

In order to characterize the af interlayer ordering further, PNR measurements of the $\left[\mathrm{Co}_{2} \mathrm{MnGe} / \mathrm{V}\right]$ multilayers with the growth induced magnetic easy axis of the multilayers parallel to the small neutron guiding field were taken (figure 23). Figure 23 presents the intensity measured for the spin channels $((+,+)$, $(-,-)$ and $(+,-))$, only the non spin flip channels show a finite intensity. Thus the sublattice magnetization of the af lattice is pointing parallel or antiparallel to the magnetic field. The first order structural peak in figure 23 has the same intensity for the $(+,+)$ and the $(-,-)$ channel. This proves that the magnetic interlayer superstructure has no ferromagnetic component which could occur e.g. by some canting of the af coupled layers. From this we can conclude that the af interlayer structure of the multilayer is well defined.

The measurement of PNR scans as a function of an applied field is shown in figure 24. The af peak intensity is rapidly suppressed at higher magnetic fields, simultaneously the splitting of the $(-,-)$ and the $(+,+)$ scattering intensities increases until at the ferromagnetic saturation field there is only a finite scattering intensity in the $(+,+)$ channel. The ferromagnetic saturation field derived from 


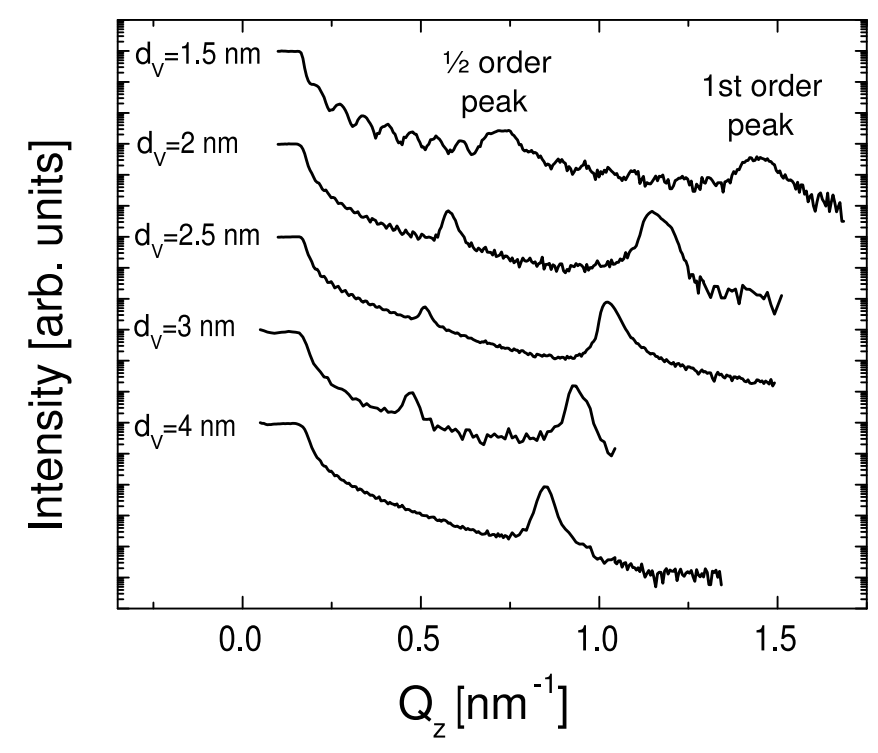

Figure 22: Specular unpolarized neutron reflectivity scans of $\left[\mathrm{Co}_{2} \mathrm{MnGe}(3 \mathrm{~nm}) / \mathrm{V}(d)\right]_{n}$ multilayers. The $\mathrm{V}$ layer thickness $d_{V}$ is given in the figure. For $d_{V}=1.5 \mathrm{~nm}$ the number of bilayers is $n=20$; in all other cases $n=50$. 


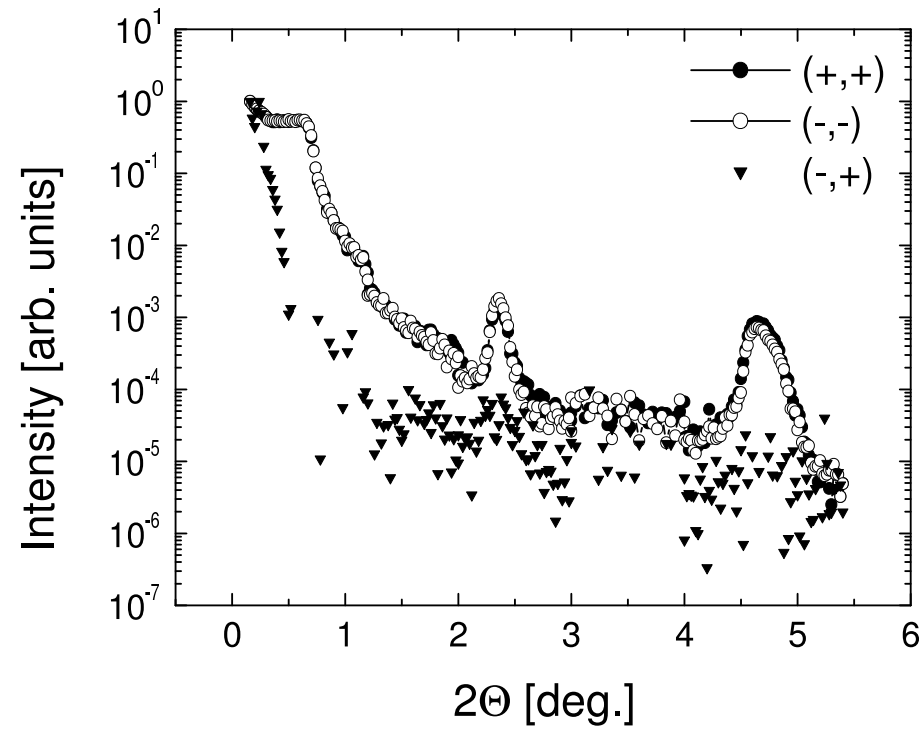

Figure 23: Specular polarized neutron reflectivity scans for non spin flip $((+,+)$ and $(-,-))$ and for spin flip $(-,+)$ channels of the multilayer $\left[\mathrm{Co}_{2} \mathrm{MnGe}(3 \mathrm{~nm}) / \mathrm{V}(2 \mathrm{~nm})\right]_{50}$.

these measurements is 50 Oe at $200 \mathrm{~K}$ and 300 Oe at $4 \mathrm{~K}$. Thus the interlayer coupling interaction in this system is very weak. This holds for all af ordered multilayers of the $\left[\mathrm{Co}_{2} \mathrm{MnGe} / \mathrm{V}\right]$ system.

There are three additional features in figure 24 worth mentioning: First, at intermediate fields there is no observable spin flip scattering i.e. the magnetization reversal of the af state takes place by domain wall movements within the single ferromagnetic layers and not by coherent rotations [84]. Second, the af structure is irreversibly lost when magnetizing the sample to the ferromagnetic saturation field at low temperatures. Only at high temperatures above about $100 \mathrm{~K}$ the af order is partly restored. This is also clearly observed in the series of magnetic hysteresis curves measured for $T<T_{N}$ shown in figure 13a. At low temperatures one observes typical ferromagnetic hysteresis loops with a remanent magnetization of up to $90 \%$ of the saturation magnetization. Third, we note that in ferromagnetic saturation in figure 24 the intensity in the $(+,+)$ channel vanishes completely i.e the neutron reflected from the multilayer are completely spin polarized in the up direction. This originates from the fact that in the $\mathrm{Co}_{2} \mathrm{MnGe}$ layer the magnetic and structural neutron scattering cross section have the same magnitude so that for one spin direction in ferromagnetic saturation the magnetic and the structural scattering length just cancel.

The temperature dependence of the af peak intensity as measured after cooling in zero-field and after cooling in 1000 Oe and then switching off the field at 


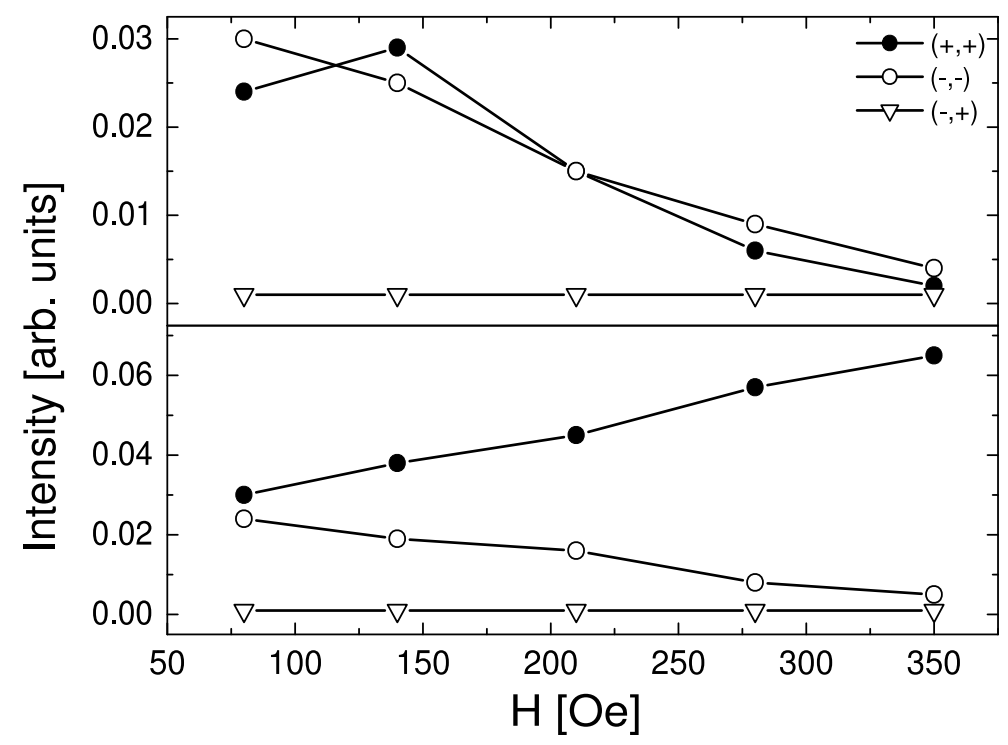

Figure 24: Field dependence of the af peak intensity (upper panel) and 1st order structural peak (lower panel) for the spin flip $(-,+)$ and non spin flip $((+,+)$ and $(-,-))$ channels for the multilayer $\left[\mathrm{Co}_{2} \mathrm{MnGe}(3 \mathrm{~nm}) / \mathrm{V}(2 \mathrm{~nm})\right]_{50}$.

the measuring temperature is displayed in figure 25. After zero-field cooling the af peak intensity develops below $270 \mathrm{~K}$ in a phase transition like fashion, reaches a maximum at about $200 \mathrm{~K}$ and decreases slightly towards lower temperatures. Cooling in a high field there is no detectable intensity below $100 \mathrm{~K}$, but approaching the phase transition at $270 \mathrm{~K}$ the af order recovers after switching off the field and close to the transition temperature the peak intensity coincides with that measured after zero-field cooling. Since the half order peak intensity is proportional to the squared sublattice magnetization in an antiferromagnet, this behaviour clearly reveals that there is a reversible antiferromagnetic phase transition at $270 \mathrm{~K}$.

The transition can also easily be detected in the dc magnetic susceptibility (figure 26a) which exhibits a peak at $270 \mathrm{~K}$, similar to a conventional bulk antiferromagnet. An antiferromagnetic phase transition in the $\left[\mathrm{Co}_{2} \mathrm{MnGe} / \mathrm{V}\right]$ system at $270 \mathrm{~K}$ is rather surprising, since from the study of the single $\mathrm{V} / \mathrm{Co}_{2} \mathrm{MnGe} / \mathrm{V}$ trilayers with the same thickness of the $\mathrm{Co}_{2} \mathrm{MnGe}$ layer we estimated a ferromagnetic ordering temperature $T_{c}$ of $600 \mathrm{~K}$ (see section 2). In multilayers coupled by antiferromagnetic IEC the Néel temperature of the af ordering between the layers and the ferromagnetic Curie temperature of the single layers should coincide. Thus the low value for $T_{N}$ provides further evidence that the af interlayer ordering in $\left[\mathrm{Co}_{2} \mathrm{MnGe} / \mathrm{V}\right]$ is not explicable by the conventional IEC mechanism.

The key for the understanding of the af interlayer magnetic ordering in 


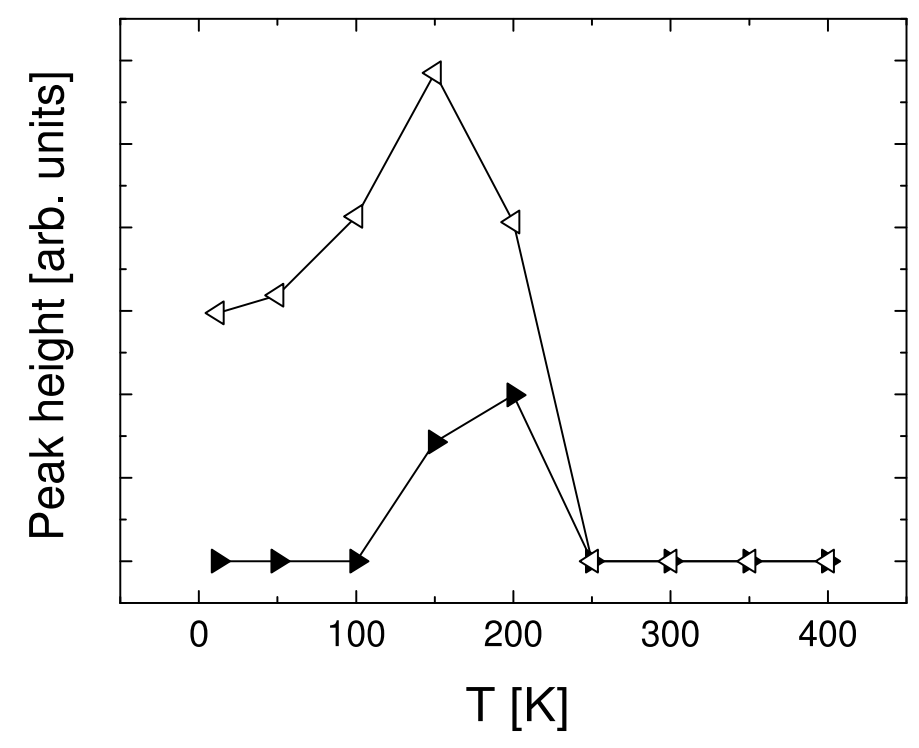

Figure 25: Temperature dependence of the af peak intensity of the multilayer $\left[\mathrm{Co}_{2} \mathrm{MnGe}(3 \mathrm{~nm}) / \mathrm{V}(3 \mathrm{~nm})\right]_{30}$ measured after field cooling (solid triangles) and zero field cooling (open triangles).

$\left[\mathrm{Co}_{2} \mathrm{MnGe} / \mathrm{V}\right]$ lies in the magnetic granular behaviour of very thin $\mathrm{Co}_{2} \mathrm{MnGe}$ layers as discussed in chapter I. By mixing and disorder at interfaces and grain boundaries the films break up into small weakly interacting ferromagnetic clusters and exhibit the typical behaviour of a small particle magnet. If the clusters are small and the cluster interactions weak, it is expected that the clusters are superparamagnetic at high temperatures 85 . In this case there is no ferromagnetic phase transition at a ferromagnetic Curie temperature $T_{c}$ but a freezing of the clusters at a superparamagnetic blocking temperature $T_{B}<T_{c}$. Actually this has not been observed in our single Co based Heusler layers from section 2 at least up to the maximum experimental temperature of $400 \mathrm{~K}$. But it may well be that at even higher temperatures a corresponding phenomenon might exist. Now, in multilayers composed of the $\mathrm{Co}_{2} \mathrm{MnGe}$ layers with the same thickness superparamagnetic behaviour and cluster blocking clearly appears below $400 \mathrm{~K}$, probably due to a change of the microstructure and the additional influence of the dipolar fields from the neighbouring layers.

The superparamagnetic behavior is shown in figure 27, where magnetization curves for the af ordered sample from figure 23 are plotted for temperatures above $T_{N}$. The $M(H)$ curves are completely reversible and saturate at fields of the order of $1 \mathrm{kOe}$ which is characteristic for a superparamagnet with a huge magnetic moment. We applied the classical formula for the superparamagnetic 

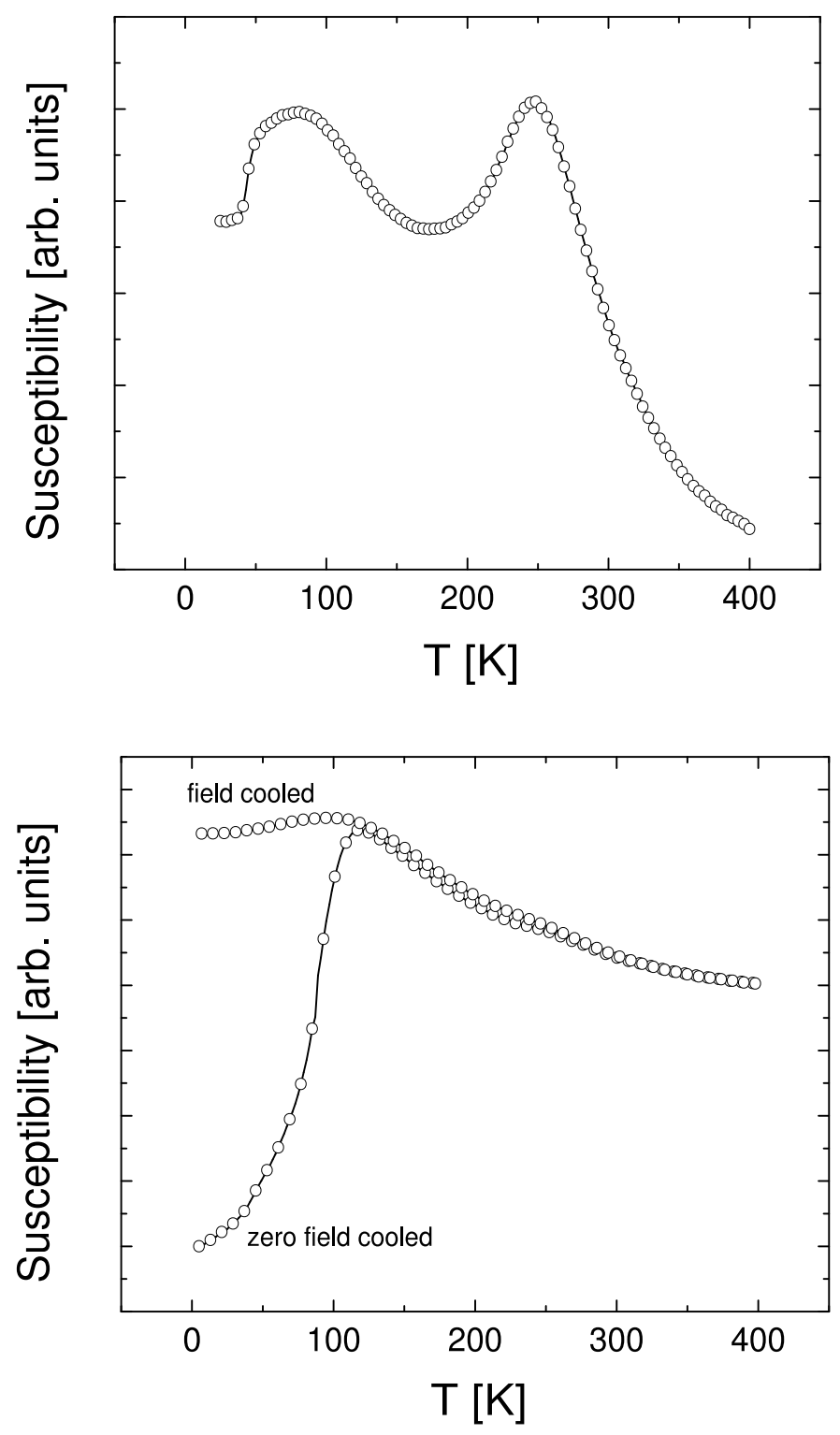

Figure 26: The dc magnetic susceptibility of the $\left[\mathrm{Co}_{2} \mathrm{MnGe}(3 \mathrm{~nm}) / \mathrm{V}(3 \mathrm{~nm})\right]_{30}$ multilayer (upper panel) and the $\left[\mathrm{Co}_{2} \mathrm{MnGe}(3 \mathrm{~nm}) / \mathrm{V}(4 \mathrm{~nm})\right]_{30}$ multilayer (lower panel). 
magnetization as a first approximation

$$
M(H, T)=N_{c} \mu_{c} \mathcal{L}\left(\frac{\mu_{c} H}{k_{b} T}\right)
$$

with the number of clusters $N_{c}$ and the cluster moment $\mu_{c}=N \mu_{B} . \mathcal{L}$ is the Langevin function. Fitting this formula to our results in figure 27 at $400 \mathrm{~K}$ we find a cluster moment of $1.6 \times 10^{5} \mu_{B}$ corresponding to a cluster with a lateral diameter of about $70 \mathrm{~nm}$.

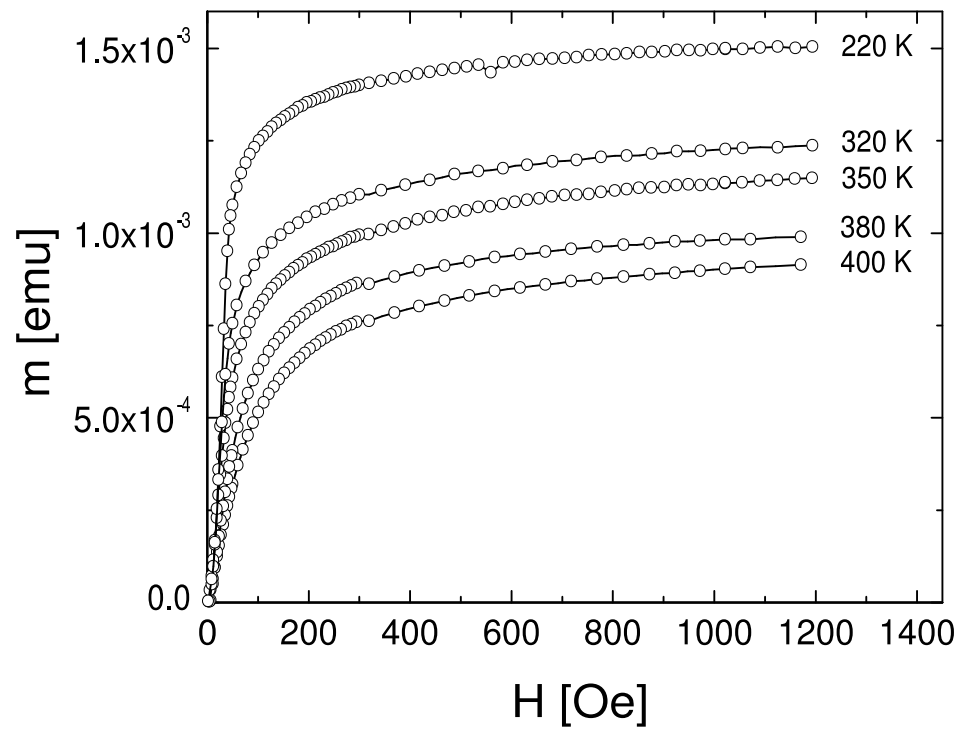

Figure 27: Magnetization curves of the af coupled multilayer $\left[\mathrm{Co}_{2} \mathrm{MnGe}(3 \mathrm{~nm}) / \mathrm{V}(3 \mathrm{~nm})\right]_{50}$ measured at different temperatures $T>T_{N}$.

These experimental findings suggest that at $T_{N}$ of the $\left[\mathrm{Co}_{2} \mathrm{MnGe} / \mathrm{V}\right]$ multilayers thermally rotating ferromagnetic clusters within each $\mathrm{Co}_{2} \mathrm{MnGe}$ layer undergo a second order phase transition with simultaneous intrta-plane ferromagnetic order and inter-plane antiferromagnetic order. This is a rather spectacular phenomenon which to the best of our knowledge has never been observed in multilayer systems before. An interesting question now arises concerning the type of magnetic order for the multilayer in figure 22 with a thickness of the $\mathrm{V}$ layer of $4 \mathrm{~nm}$, which obviously does not exhibit af long range order. The answer is given in figure 26 $\mathrm{b}$, where the zero field cooled and the field cooled magnetization of this sample is compared. There is an onset of a strong magnetic irreversibility at about $150 \mathrm{~K}$, reminiscent of a cluster blocking or a cluster glass magnetic ordering [85]. Actually in the hysteresis loops measured at low temperatures for this sample one finds features consistent with this type of transition, namely a temperature dependence of the coercive force and a magnetic 
remanence following a power law behaviour $H_{c} \sim T^{-a}$.

The magnetic order in the $\left[\mathrm{Co}_{2} \mathrm{MnGe} / \mathrm{V}\right]$ multilayer system for different thickness of the $\mathrm{V}$ layer is summarized in a magnetic phase diagram in figure 28. There is an antiferromagnetic range for $d_{V} \leq 3 \mathrm{~nm}$ with the Néel temperature increasing with decreasing $d_{V}$ up to $T_{N}=380 \mathrm{~K}$ for $d_{V}=1.5 \mathrm{~nm}$. Then a range with cluster blocking or cluster glass freezing follows with the magnetic transition temperature nearly independent of $d_{V}$ and extending up to the maximum thickness we have studied $d_{V}=10 \mathrm{~nm}$.

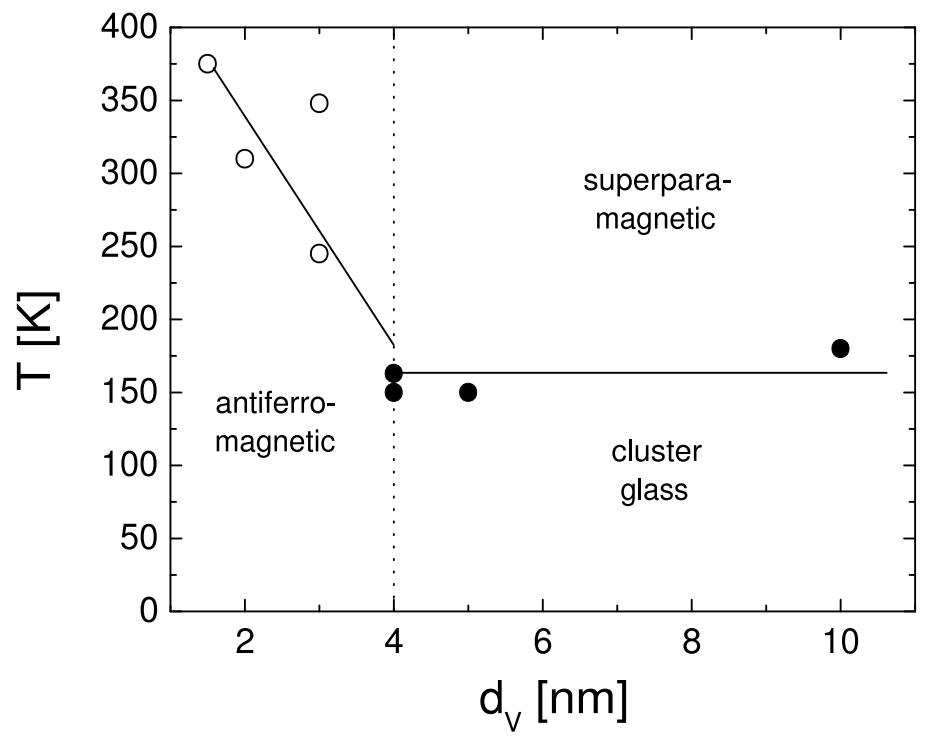

Figure 28: Magnetic phase diagram for multilayers $\left[\mathrm{Co}_{2} \mathrm{MnGe}(3 \mathrm{~nm}) / \mathrm{V}(d)\right]_{50}$ with different vanadium thickness $d_{V}$. There is an antiferromagnetic range for $d_{V} \leq 3 \mathrm{~nm}$ and a range with cluster blocking for $d_{V}>4 \mathrm{~nm}$.

Concerning the microscopic origin of the ordering phenomenon observed in the $\left[\mathrm{Co}_{2} \mathrm{MnGe} / \mathrm{V}\right]$ multilayers here, we would like to emphasize that the IEC coupling plays no essential role. It is either too weak or cancels out because of thickness fluctuations. The remaining other candidate which can produce an interlayer coupling and af long range order are interlayer magnetic dipolar fields. Finite dipolar stray fields at the surface of each single $\mathrm{Co}_{2} \mathrm{MnGe}$ layer are inevitably present, since the layers possess an internal granular magnetic structure. Topologically the magnetic surface is rough on the length scale of the dimension of the clusters i.e. on a scale of about several $10 \mathrm{~nm}$.

Theoretical model calculations show that the dipolar coupling field seen by the neighbouring layers depends on the topological structure of the surface and can be ferromagnetic or antiferromagnetic, depending on details of the roughness [86]. Quantitative calculations with a surface structure mimicking typical thin 
film systems lead to an estimation of a coupling field strength of the order of 100 Oe i.e. the order of magnitude which we observed here. Experimentally there is the well known Néel type af coupling [87] between thin films separated by a very thin nonmagnetic interlayer, which is a disturbing factor in some spin valve devices [88. Originally this coupling, which is of dipolar origin, has been proposed by Néel [87. Dipolar fields can also produce antiferromagnetic long range order in multilayers, as has been shown for $[\mathrm{Co} / \mathrm{Cu}]$ multilayers with a thickness of the $\mathrm{Cu}$ layer of $6 \mathrm{~nm}$ [89]. But in this system the af long range order is only observed in the virgin state and is lost irreversibly after once magnetizing the sample. In $[\mathrm{Nb} / \mathrm{Fe}]$ multilayers the existence of af interlayer dipolar interaction has also been shown experimentally [90].

In summary of this section, we find an interesting novel magnetic ordering phenomenon in the $\left[\mathrm{Co}_{2} \mathrm{MnGe} / \mathrm{V}\right]$ multilayers which is directly related to the granular magnetic microstructure of the Co based Heusler thin films in the limit of very small thickness. The antiferromagnetic order is very subtle and we have only observed it in $\left[\mathrm{Co}_{2} \mathrm{MnGe} / \mathrm{V}\right]$ multilayers with a thickness of the Heusler layer of $3 \mathrm{~nm}$, although magnetic granularity is a common feature of all Co based Heusler phases with a comparable thickness. However, when combined into multilayers all other systems we have studied remain ferromagnetic without the cluster blocking features and the af long range order characteristic for the $\left[\mathrm{Co}_{2} \mathrm{MnGe} / \mathrm{V}\right]$ multilayers. Thus in this sense the phenomenon is rather unique for $\left[\mathrm{Co}_{2} \mathrm{MnGe} / \mathrm{V}\right]$ multilayers.

\section{Magnetotransport Properties}

For possible technical applications such as GMR devices based on the fully spin polarized Heusler compounds, the electrical transport properties are of utmost importance. Thus we summarize here our results on the electrical conductivity, the Hall conductivity and the magnetoresistance of the pure Heusler films and the Heusler multilayers. The electrical conductivity was measured by 4 point technique with silver painted electrical contacts, for the measurement of the Hall conductivity the films were patterned into the conventional Hall bar geometry with five contacts produced by ion beam etching. Figure 29 displays the electrical resistivity versus temperature for the optimized 100-nm thick pure films of the Co based Heusler alloys. The residual resistivity is rather high, especially for the $\mathrm{Co}_{2} \mathrm{MnSi}$ film (taken from reference [67]) with a residual resistivity ratio $(R R R)$ (defined as the ratio of the room temperature resistivity and the resistivity at $4 \mathrm{~K}$ ) of $R R R=1.07$. The $\mathrm{Co}_{2} \mathrm{MnSn}$ and the $\mathrm{Co}_{2} \mathrm{MnGe}$ films have a higher electrical conductivity and a higher $R R R\left(R R R=1.15\right.$ for the $\mathrm{Co}_{2} \mathrm{MnSn}$ phase and $R R R=1.55$ for the $\mathrm{Co}_{2} \mathrm{MnGe}$ phase). Actually the $R R R$ of 1.55 for $\mathrm{Co}_{2} \mathrm{MnGe}$ is the highest value reported for thin films of the Co based fully spin polarized Heusler compounds in the literature until now.

It holds generally true for thin films of the Co based Heusler compounds that there is strong electron scattering at defects such as grain boundaries, antisite defects, interstitials and voids. We determined the Hall coefficient $R_{0}$ 


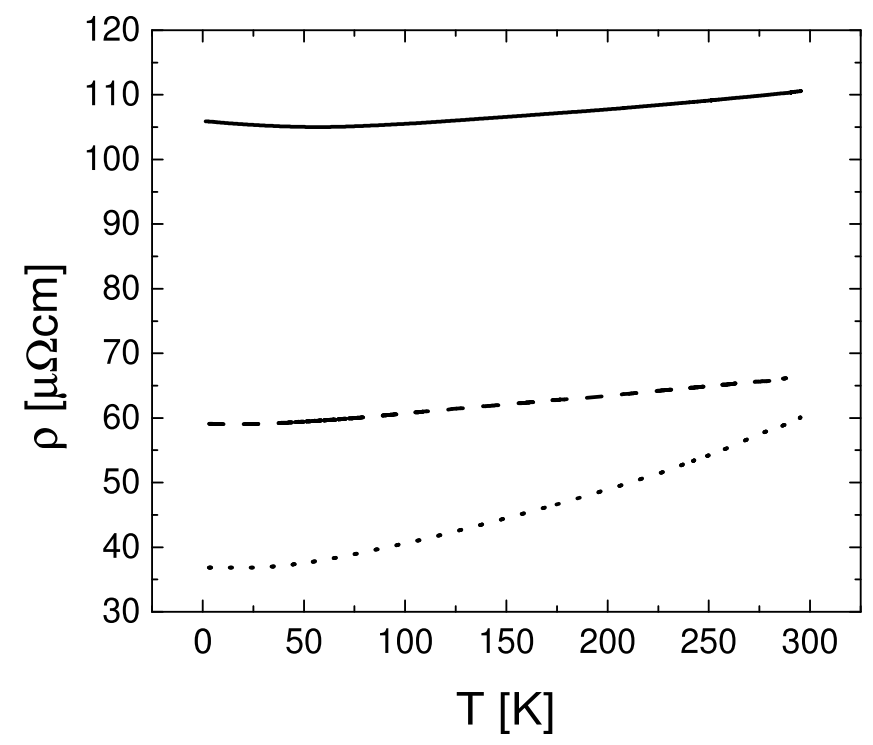

Figure 29: Electrical resistivity versus temperature for $\mathrm{V}(3 \mathrm{~nm}) / \mathrm{Co}_{2} \mathrm{MnSi}(100 \mathrm{~nm})$ (solid line), $\mathrm{Au}(3 \mathrm{~nm}) / \mathrm{Co}_{2} \mathrm{MnSn}(100 \mathrm{~nm})$ (dashed line) and $\mathrm{V}(3 \mathrm{~nm}) / \mathrm{Co}_{2} \mathrm{MnGe}(100 \mathrm{~nm})$ (dotted line).

by fitting the Hall voltage measured up to a magnetic field $\mu_{0} H=3 \mathrm{~T}$ by the formula $U_{H}=R_{0} H+R_{H} 4 \pi M$ with the magnetization $M$ and the anomalous Hall coefficient $R_{0}$ and derived $R_{0}=2 \times 10^{-10} \mathrm{~m}^{3} / \mathrm{As}$ for the $\mathrm{Co}_{2} \mathrm{MnSi}$ phase and $R_{0}=2.2 \times 10^{-10} \mathrm{~m}^{3} /$ As for the $\mathrm{Co}_{2} \mathrm{MnSn}$ phase. Combining with the electrical conductivity and assuming parabolic conduction bands we estimate an electron mean free path $l_{e}$ at $4 \mathrm{~K}$ of $l_{e}=1.3 \mathrm{~nm}$ for the $\mathrm{Co}_{2} \mathrm{MnSi}$ phase and $2.2 \mathrm{~nm}$ for the $\mathrm{Co}_{2} \mathrm{MnSn}$ phase. Assuming that for the $\mathrm{Co}_{2} \mathrm{MnGe}$ layer we have a similar value for $R_{0}$ we estimate $l_{e}=5.5 \mathrm{~nm}$ for this thin film in figure 29. These remarkably low values for $l_{e}$ stresses the strong defect scattering in these compounds.

In figure 30 we show resistivity measurements of several multilayers from section 3. We find high absolute values for the resistivity, the resistivity ratio is below $R R R=1.1$ for $\mathrm{Co}_{2} \mathrm{MnGe}$ and $\mathrm{Co}_{2} \mathrm{MnSn}$ with $\mathrm{V}$ and $\mathrm{Au}$ interlayers. In these multilayers $l_{e}$ is very short namely of the order of $1 \mathrm{~nm}$. If one inspects the resistivity curves in figures 29 and 30 in more detail, one observes a shallow minimum in $\rho(\mathrm{T})$ at temperatures between $20 \mathrm{~K}$ and $50 \mathrm{~K}$ for all samples with an $R R R$ below about $R R R=1.2$. This is a common feature for the Co based Heusler thin films. The low temperature resistivity $\rho(\mathrm{T})$ is plotted on an enlarged scale in figure 31. The amplitudes of the resistivity upturn towards low temperatures and the temperature of the resistivity minimum increases with increasing defect scattering, thus the low temperature anomaly is clearly related 


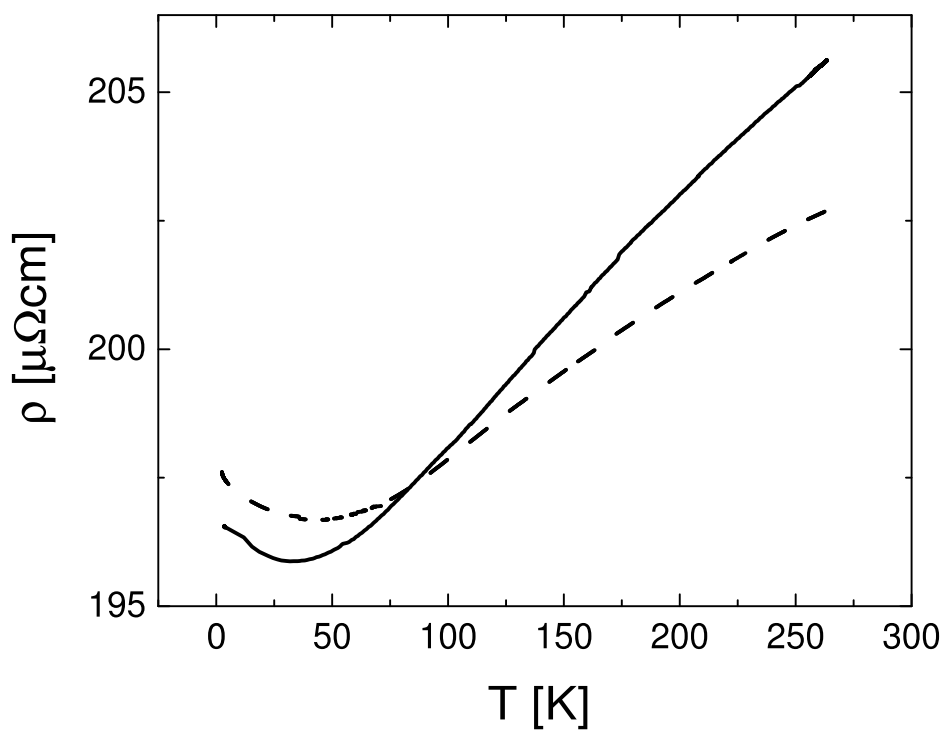

Figure 30: Resistivity versus temperature for the multilayers $\left[\mathrm{Co}_{2} \operatorname{MnSn}(3 \mathrm{~nm}) / \mathrm{V}(3 \mathrm{~nm})\right]_{30}$ (dashed line) and $\left[\mathrm{Co}_{2} \mathrm{MnGe}(3 \mathrm{~nm}) / \mathrm{V}(3 \mathrm{~nm})\right]_{50}$ (straight line). 


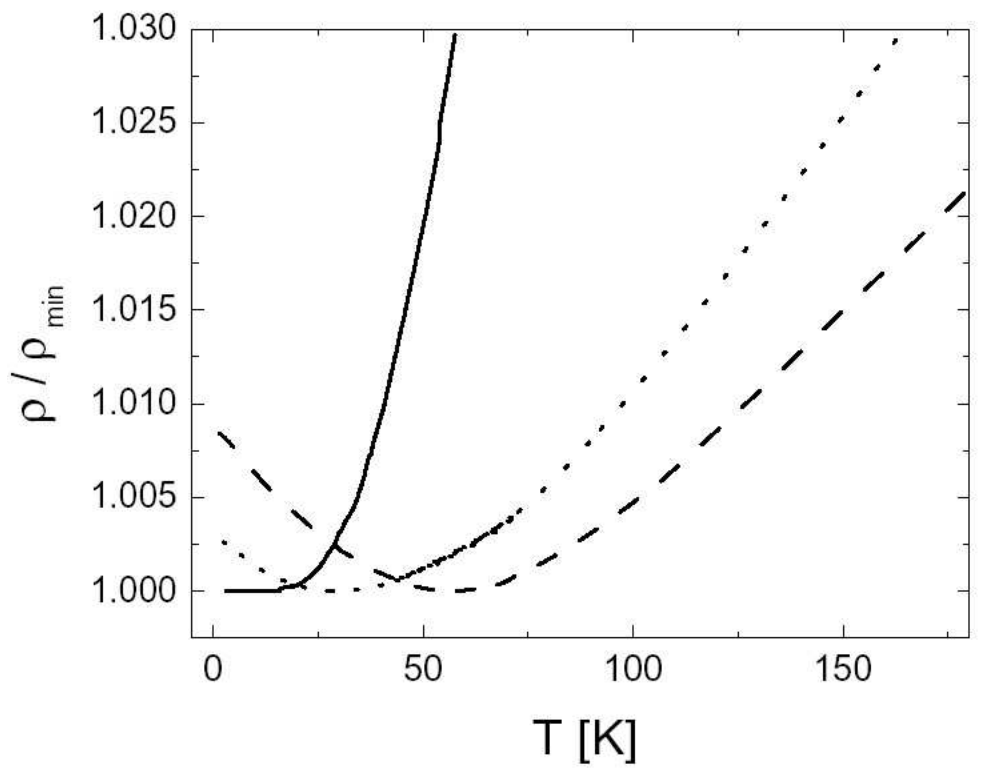

Figure 31: Low temperature resistivity of $\mathrm{Co}_{2} \mathrm{MnSi}$ (dashed line), $\mathrm{Co}_{2} \mathrm{MnSn}$ (dotted line) and $\mathrm{Co}_{2} \mathrm{MnGe}$ (straight line) films. The $\mathrm{Co}_{2} \mathrm{MnSi}$ and the $\mathrm{Co}_{2} \mathrm{MnSn}$ phase show an upturn of the resistivity towards lower temperatures. 


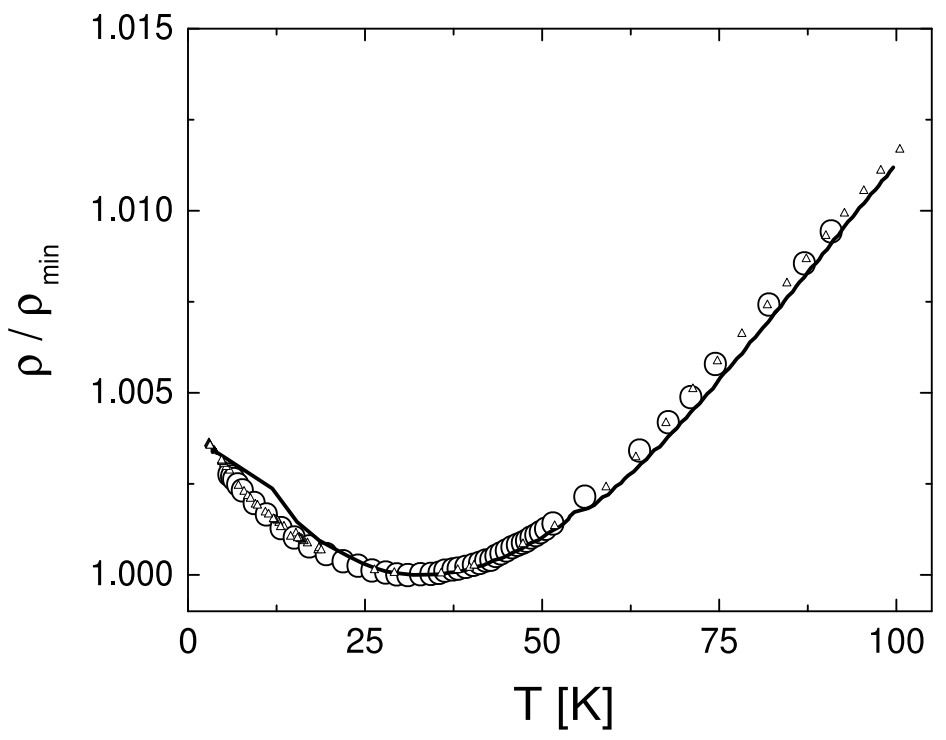

Figure 32: Electrical resistivity versus temperature for the multilayer $\left[\mathrm{Co}_{2} \mathrm{MnGe}(3 \mathrm{~nm}) / \mathrm{Au}(3 \mathrm{~nm})\right]_{50}$ measured in zero field (straight line) and with an applied field of $4 \mathrm{~T}$ parallel to the film (open triangles) and perpendicular to it (open dots).

to disorder. A low temperature upturn in $\rho(\mathrm{T})$ is well known for metallic thin films and is usually associated with the Kondo effect 91 or weak localization 92]. But these effects seem not very plausible here, since in a strong ferromagnet Kondo scattering is hardly possible and measurable weak localization effects are only expected in two dimensional metals. There is a third classical effect leading to a low temperature upturn in $\rho(\mathrm{T})$ which is less well known. This is a renormalization of the electronic density of states at the Fermi level in metals with strong disorder [93]. This essentially is an electronic correlation effect and becomes relevant if the scattering of the conduction electrons is strong and $l_{e}$ small. This renormalization effect has experimentally been observed in amorphous metals 94 and gives a plausible explanation for the low temperature anomaly in $\rho(\mathrm{T})$ of the Heusler films, too.

A measurement which strongly supports this hypothesis is shown in figure 32. where for the example of a $\left[\mathrm{Co}_{2} \mathrm{MnSn} / \mathrm{Au}\right]$ multilayer it is shown that a strong magnetic field of $4 \mathrm{~T}$ applied in the direction parallel and perpendicular to the film does not change the low temperature upturn in $\rho(\mathrm{T})$. This is expected for the renormalization effect, whereas for Kondo scattering and also in the case of weak localization the upturn should be suppressed.

Coming to the magnetoresistance (MR) of the pure thin films and the multilayers we first discuss the high field MR i.e. the resistance for fields above the 


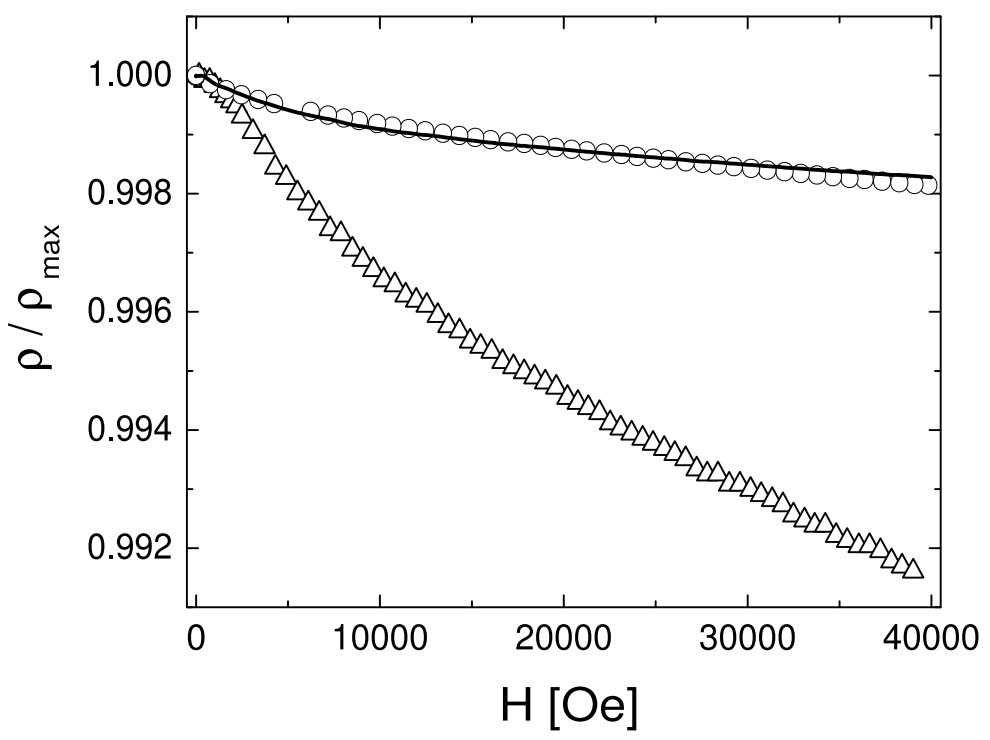

Figure 33: High field magnetoresistance measured at $4 \mathrm{~K}$ for $\mathrm{V}(3 \mathrm{~nm}) / \mathrm{Co}_{2} \mathrm{MnSi}(100 \mathrm{~nm})$ (straight line), $\mathrm{Au}(3 \mathrm{~nm}) / \mathrm{Co}_{2} \mathrm{MnSn}(100 \mathrm{~nm})$ (open dots) and $\mathrm{V}(3 \mathrm{~nm}) / \mathrm{Co}_{2} \mathrm{MnGe}(100 \mathrm{~nm})$ (open triangles). 


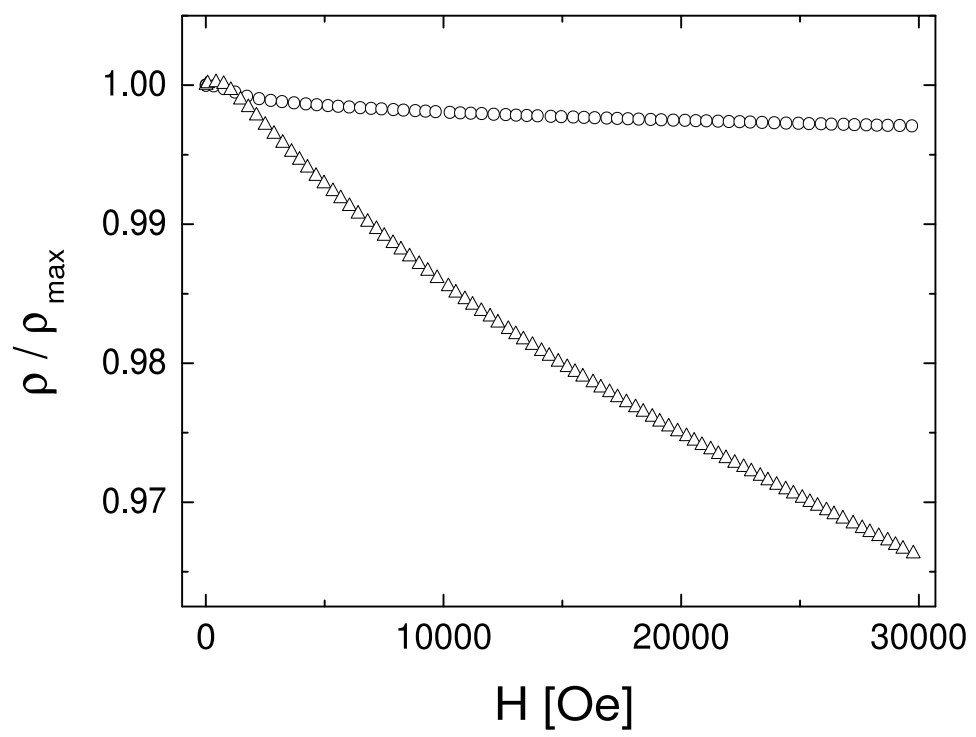

Figure 34: High field magnetoresistance measured at $4 \mathrm{~K}$ for the multilayers $\left[\mathrm{Co}_{2} \mathrm{MnSn}(3 \mathrm{~nm}) / \mathrm{V}(3 \mathrm{~nm})\right]_{30}$ (open dots) and $\left[\mathrm{Co}_{2} \mathrm{MnSn}(3 \mathrm{~nm}) / \mathrm{Au}(3 \mathrm{~nm})\right]_{30}$ (straight line).

ferromagnetic saturation field where the anisotropic magnetoresitance (AMR) and the Giant magnetoresistance (GMR) play no role. This high field magnetoresistance measured at $4 \mathrm{~K}$ is plotted for the pure Heusler films in figure 33 and for several multilayers in figure 34. The high field magnetoresistance for the pure Heusler films is negative and isotropic. The total decrease of the resistance for fields up to $4 \mathrm{~T}$ is about $0.16 \%$ for the $\mathrm{Co}_{2} \mathrm{MnSi}$ and the $\mathrm{Co}_{2} \mathrm{MnSn}$ phase and definitely higher, namely $0.8 \%$ for the $\mathrm{Co}_{2} \mathrm{MnGe}$ single crystalline film. We interpret the high field MR as a reduction of spin disorder scattering in a ferromagnet with non perfect ferromagnetic alignment of all spins. This interpretation is supported by the results of the high field MR on the multilayers in figure 34. For the $\left[\mathrm{Co}_{2} \mathrm{MnSn} / \mathrm{Au}\right]$ multilayer where the exchange bias effect discussed in section 3.2 gives clear evidence for the existence of magnetic frustration and canted spins at the interfaces, we find an unusually large MR value with an amplitude of $3 \%$ up to a field of $3 \mathrm{~T}$, whereas for the $\left[\mathrm{Co}_{2} \mathrm{MnGe} / \mathrm{V}\right]$ multilayer showing no indication of magnetic frustration the MR is only $0.3 \%$.

In the low field MR measurements i.e. the MR for fields up to the coercive force $H_{c}$ we observed the standard AMR of a ferromagnetic film, however with a very small relative difference of the resistance for the field applied parallel and perpendicular to the current of the order $\Delta \rho / \rho=4 \times 10^{-4}$. For the multilayers the AMR is even smaller i.e. below the experimental resolution limit for $\Delta \rho / \rho$ of about $10^{-5}$. 


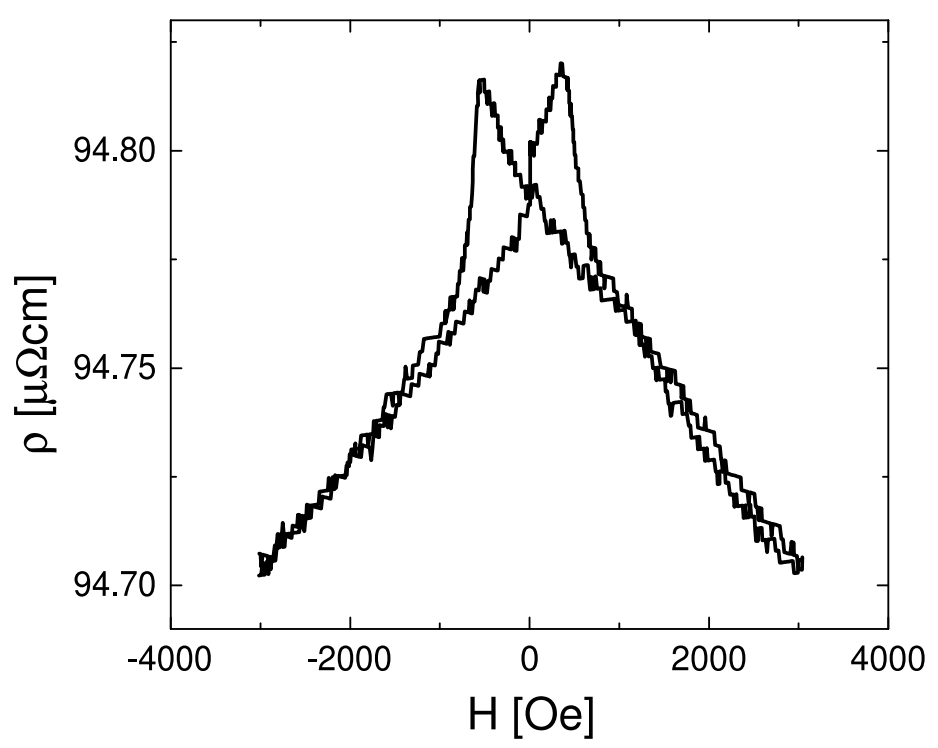

Figure 35: Low field magnetoresistance measured at $4 \mathrm{~K}$ of a $\mathrm{Co}_{2} \mathrm{MnGe}(3 \mathrm{~nm}) / \mathrm{V}(3 \mathrm{~nm}) / \mathrm{Co}_{2} \mathrm{MnGe}(3 \mathrm{~nm}) / \mathrm{CoO}(2 \mathrm{~nm})$ trilayer.

In figures 35 and 36 we present low field MR measurements related to the GMR effect. Figure 35 displays the $\mathrm{MR}$ at $4 \mathrm{~K}$ of a $\mathrm{Co}_{2} \mathrm{MnGe} / \mathrm{V} / \mathrm{Co}_{2} \mathrm{MnGe} / \mathrm{CoO}$ trilayer, where an exchange bias with $\mathrm{CoO}$ is used to pin the magnetization direction of the upper $\mathrm{Co}_{2} \mathrm{MnGe}$ layer [77]. One finds a small jump in the MR at $H_{c}$ with an amplitude $\Delta \rho / \rho=5 \times 10^{-4}$ which could be associated with a GMR effect. In figure 36 we show the low field $\mathrm{MR}$ of a $\left[\mathrm{Co}_{2} \mathrm{MnGe} / \mathrm{V}\right]$ multilayer measured at $4 \mathrm{~K}$ and starting from the antiferromagnetically ordered state of the multilayer obtained after zero field cooling (see section 3.3). Driving the antiferromagnetically coupled $\mathrm{Co}_{2} \mathrm{MnGe}$ layers to ferromagnetic saturation and returning to zero field, gives an irreversible change of the MR $\Delta \rho / \rho=5 \times 10^{-4}$, which is the upper limit for the GMR effect.

This chapter should demonstrate the difficulties one encounters when trying to use the fully spin polarized Co based Heusler alloys in magnetotransport applications such as GMR or AMR. The electrical conductivity and the electron mean free path are small, pointing towards the existence of lattice defects with a high conduction electron scattering efficiency. Our results suggest that these are the same antisite defects in the $\mathrm{L} 2_{1}$ structure which have the tendency to destroy the half metalicity. The rather low values for the GMR in the spin valve device and the antiferromagnetically coupled multilayers are not really surprising since there are three detrimental factors which acting together are able to nearly eliminate the GMR effect: First,non ferromagnetic interlayers are present introducing strong spin independent scattering thereby reducing the GMR. Sec- 


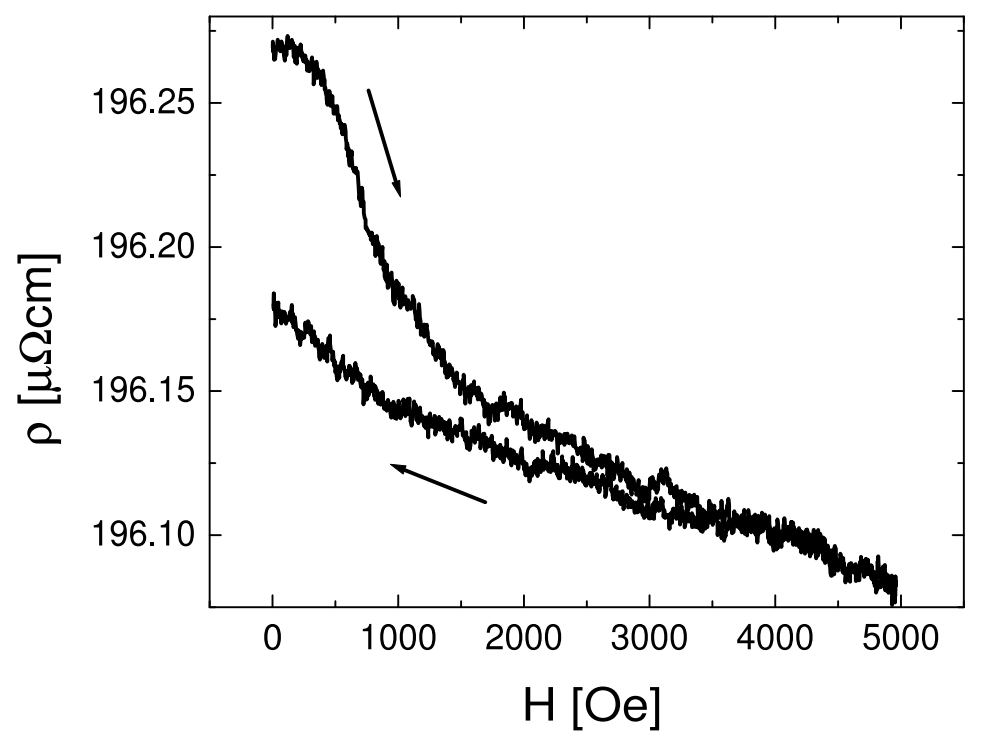

Figure 36: Low field magnetoresistance measured at $4 \mathrm{~K}$ of a $\left[\mathrm{Co}_{2} \mathrm{MnGe}(3 \mathrm{~nm}) / \mathrm{V}(3 \mathrm{~nm})\right]_{50}$ multilayer, starting at the antiferromagnetic state after zero field cooling (arrows indicate the field cycle). 
ond, for the observation of a sizable GMR effect, the conduction electron mean free path should be larger than the double layer thickness [55], which is certainly not fulfilled in our samples. Last but not least, the high spin polarization expected for the ideal Co based Heusler compounds is most certainly lost in very thin Co based Heusler layers with a saturation magnetization of typically only $50 \%$ of bulk samples.

\section{Summary and Conclusions}

We have shown that thick single films of the Heusler compounds $\mathrm{Co}_{2} \mathrm{MnGe}$ and $\mathrm{Co}_{2} \mathrm{MnSn}$ can be grown with a saturation magnetization and structural quality comparable to bulk samples. However, when decreasing the thickness to the order of $3 \mathrm{~nm}$ the films show a definitely lower value of the saturation magnetization and exhibit properties reminiscent of small particle ferromagnets. We interpret this behaviour by a magnetic decoupling of the Heusler films at the grain boundaries of the very small grains forming the film. Structural disorder and a high concentration of antisite defects at the grain boundaries weaken the ferromagnetism drastically.

As we have shown by the XMCD measurements on the Mn and the Co $L_{2,3}$ absorption edges, it is only the Mn atom in the Heusler compounds which reduces the saturation magnetization. Theoretical model calculations suggest that this originates from an antiparallel spin orientation of antisite Mn spin rather than from a loss of the atomic magnetic moment of Mn. In the multilayers a similar phenomenon occurs at the interfaces of the Heusler alloys with other metals. For a thickness range of about 0.5 to $0.7 \mathrm{~nm}$ at the interfaces the Heusler alloys are only weakly ferromagnetic or exhibit a spin glass type of order. The exchange bias shift of the hysteresis loops observed at low temperatures gives clear evidence for this.

In $\left[\mathrm{Co}_{2} \mathrm{MnGe} / \mathrm{V}\right]$ multilayers we have detected a peculiar antiferromagnetic interlayer magnetic ordering with a well defined Néel temperature far below the ferromagnetic Curie temperature of a single Heusler layer of the same thickness. The antiferromagnetic ordering exists in the thickness range of the $\mathrm{V}$ interlayer between $1 \mathrm{~nm}$ and $3 \mathrm{~nm}$, very unlike the antiferromagnetic interlayer order in multilayer systems coupled by interlayer exchange mechanism. The antiferromagnetic order is directly related to the granular ferromagnetic structure of very thin Heusler layers. The small ferromagnetic particles defined by the weak magnetic coupling at the grain boundaries exhibit superparamagnetic behaviour above the Néel temperature. The interlayer dipolar interactions between the superparamagnetic particles cause a reversible magnetic phase transition with antiferromagnetic order between the layers and ferromagnetic order within the layers at a well defined Néel temperature.

Finally, the magnetotransport properties revealed that the electron mean free path in the single layers and in the multilayers is very small, indicative of the presence of large amounts of very effective electron scattering centers. Si-

multaneously there exists an isotropic negative spin disorder magnetoresistance 
up to very high fields, which shows that the magnetic ground state is not a perfect ferromagnet but possesses abundant magnetic defects. We think that the main source of defects responsible for the small mean free path and the magnetic disorder scattering are the antisite defects in the $\mathrm{L} 2_{1}$ structure.

Coming back to the issue discussed in the introduction, namely the perspective of the Heusler compounds as full spin polarized ferromagnetic layers in magnetoelectronic devices, our results indicate the severe difficulties one inevitably encounters. The constraints in the preparation of thin film heterostuctures in many cases prohibit the optimum preparation conditions for the Heusler phase thus rendering it difficult to get the $\mathrm{L} 22_{1}$ structure with a high degree of atomic order. At the interfaces additional problems arise since they tend to be strongly disordered and weakly ferromagnetic. What one ideally would need to overcome these problems is a Heusler phase with a more robust spin polarization at the Fermi level which principally might already exist among the many new Heusler half metals which have been predicted theoretically. In any case, the evolving field of magnetoelectronics motivated the magnetism community to extend the experimental activities in thin film preparation towards complex intermetallic ternary alloys, which is certainly much more difficult than the preparation of simple films but offers new and interesting perspectives for basic research and applications.

Acknowledgement The authors want to thank the DFG for financial support of this work within the SFB 491 Bochum/Duisburg

\section{References}

[1] F. Heusler: Verh. Dtsch. Phys. Ges. 5, 219 (1903)

[2] P.J. Webster and K.R.A. Ziebeck. In: Alloys and Compounds of d-Elements with Main Group Elements. Part 2., Landolt-Börnstein, New Series, Group III, vol 19c, ed by H.R.J. Wijn, (Springer, Berlin 1988) pp 75-184

[3] J. Soltys: Phys. Stat, Sol. (a) 66, 485 (1981)

[4] A. Zheludev, S. M. Shapiro, P. Wochner, A. Schwartz, M. Wall, and L. E. Tanner: Phys. Rev. B 51, 113109 (1995)

[5] R. Kosubski, and J. Soltys: J Mat. Science 17, 1441 (1982)

[6] K.R.A. Ziebeck, and P.J. Webster: J. Phys. F 5, 1756 (1975)

[7] P.J. Webster, and R.S. Tebbel: J. Appl. Phys 39, 471 (1968)

[8] P.J. Webster: J. Phys. Chem. Sol. 32, 1221 (1971)

[9] K.H.J. Buschow, and P.G. van Engen: J. Magn. Mag. Mat. 25, 90 (1983)

[10] J. Kübler, A.R. Williams, and C.B. Sommers: Phys. Rev. B 28, 1745 (1983) 
[11] K.H.J. Buschow, and M. Erman: J. Magn. Magn. Mat. 30, 374 (1983)

[12] J. Pierre, R.V. Skolozdra, and Yu. V. Stadnyk: J. Magn. Magn. Mat. 128, $93(1993)$

[13] Y. Nishino, M. Kato, S. Asano, K. Soda, M. Hayasaki, and U. Mizutani: Phys. Rev. Lett. 79, 1909 (1997)

[14] A. Siebarski, M. B. Maple, E. J. Freeman et al.: Phys. Rev. B 62, 3296 (2000)

[15] H. Okamura, J. Kawahara, T. Nanba et al.: Phys. Rev. Lett. 84, 3674 (2000)

[16] R. de Groot and P. van Engen: Phys. Rev. Lett. 50, 2024 (1983)

[17] S. Fujii, S. Sugimura, S. Ispida, and S. Asano: J. Phys.: Cond. Matter 2, $8583(1990)$

[18] S. Ishida, S. Kashiwagi, S. Fujii, and S. Asano: Physica B 210, 140 (1995)

[19] S. Ishida, S. Fujii, S. Kashiwagi, and S. Asano: J. Phys. Soc. Jpn. 64, 2154 (1995)

[20] K.E.H.M Hansen, P.R. Mijnarends, L.P.L.M. Rabou, and K.H.J. Buschow: Phys. Rev. B 42, 1533 (1990)

[21] M.J. Otto, R.A.M. van Woerden, P.J. van der Valk, J. Wijngaard, C.F. van Bruggen, and C. Haas: J. Phys.: Cond. Matter 1, 2351 (1989)

[22] C. Hordequin, J. Pierre, and R. Currat: J. Magn. Magn. Mat. 162, 75 (1996)

[23] R.J. Soulen, J.M. Byers, M.S. Osofsky, B. Nadgony et al.: Science 282, 85 (1998)

[24] P.J. Brown, K.U. Neumann, P.J. Webster, and K.R.A. Ziebeck: J. Phys.: Cond. Matter 12, 1827 (2000)

[25] L. Ritchie, G. Xiao, Y. Ji, T.Y. Chen, M. Zhang et al.: Phys. Rev. B 68, $104430(2003)$

[26] D. Ristoiu, J. P. Nozieres, C. N. Borca, T. Komesu, H.-K. Jeong, and P. A. Dowben: Europhys. Lett. 49, 624 (2000)

[27] W. Zhu, B. Sinkovic, E. Vescovo, C. Tanaka, and J. S.Moodera: Phys. Rev. B 64, 060403 (2001)

[28] G.A. Prinz: Science282, 1660 (1998)

[29] D. Awschalom and J. Kikkawa: Physics Today 52, 33 (June 1999) 
[30] G. Schmidt, D. Ferrand, L.W. Molenkamp, A.T. Filip, and B.J. van Wees: Phys. Rev. B 62, R4790 (2000)

[31] C. Cacho, Y. Lassailly, H.-J. Drouhin, G. Lampel, and J. Peretti: Phys. Rev. Lett. 88, 066601 (2002)

[32] J. Moodera, R. Kinder, L.T. Wong, and R. Meservey: Phys. Rev. Lett. 74, $3273(1998)$

[33] Eun-Cheol Lee, and K. J. Chang, Phys. Rev. B 69, 85205 (2004)

[34] K. Schwarz: J. Phys. F 16, L211 (1986)

[35] J.M.D. Coey, M.Viret, and S. von Molnar: Adv. Phys. 48, 167 (1999)

[36] I. Galanakis, P.H. Dederichs, and N. Papanikolaou: Phys. Rev. B 66, 174429 (2002)

[37] I. Galanakis, P.H. Dederichs, and N. Papanikolaou: Phys. Rev. B 66, $134428(2002)$

[38] C. Felser, B. Heitkamp, F. Kronast, D. Schmitz, S. Cramm, H.A. Dürr, H-J. Elmers G. H. Fecher, S. Wurmehl, T. Block, D. Valdaitsev, S. A. Nepijko, A. Gloskovskii, G. Jakob, G. Schonhense, and W. Eberhardt: J. Phys.: Condens. Matter 15, 7019 (2003)

[39] S. Picozzi, A. Continenza, and A.J. Freeman: Phys. Rev. B 69, 094423 (2004)

[40] M.P. Raphael, B. Ravel, Q. Huang, M.A. Willard, S.F. Cheng, B.N. Das, R.M. Stroud, K.M. Bussmann, J.H. Claassen, and V.G. Harris: Phys. Rev. B 66, 104429 (2002)

[41] B. Ravel, M.P. Raphael, V. G. Harris, and Q. Huang: Phys. Rev. B. 65, $164431(2002)$

[42] C. Hordequin, J.P. Nozieres, and J. Pierre: J. Magn. Magn. Mat. 183, 225 (1998)

[43] C. Tanaka, J. Novak, and J.S. Moodera: J. Appl. Phys 81, 5515 (1997)

[44] J. Schmalhorst, S. Kämmerer, M. Sacher, G. Reiss, A. Hütten, and A. Scholl: Phys. Rev. Lett. 70, 024426 (2004)

[45] I. Galanakis: J. Phys.: Condens. Matter 14, 6329 (2002)

[46] S. Ishida, T. Masaki, S. Fujii, and S. Asano: Physica B 245, 1 (1998)

[47] D. Orgassa, H. Fujiwwara, T.C. Schulthess, and W.H. Butler: Phys. Rev. B 60, 13237 (1999) 
[48] R.W. Overholser, M. Wuttig, and D.A. Neumann: Scripta Marterialia 40, 1095 (1999)

[49] G. Fritsch, V.V. Kokorin, and A. Kempf: J. Phys.:Condens. Matter 6, L107 (1994)

[50] J. Tobola and J. Pierre: J. Alloy Comp. 196, 234 (2000)

[51] E. DiMasi, M.C. Aronso, and B.R. Cole: Phys. Rev. B 47, 1430 (1993)

[52] J. Pierre, R.V. Skolozda, and M.A. Kouacou: Physica B 206, 844 (1995)

[53] J. Tobola, J. Pierre, S. Kapryzyk, R.V. Skolozda, and M.A. Kouacou: J. Magn. Magn. Mater. 159, 192 (1996)

[54] J.F. Gregg, I. Petey, E. Jougelet, and C. Dennis: J. Phys. D: Appl. Phys. 35, R121 (2002)

[55] B. Dieny: J. Magn. Magn. Mater. 136, 335 (1994)

[56] K.A. Kilian, and R.H. Victora: J. Appl. Phys. 87, 7064 (2000)

[57] V. Yu Irkhin, and M.I. Katsnelśon: Physics-Uspekhi 37, 659 (1994)

[58] W.E. Picket: Phys. Rev. Lett. 77, 3185 (1996)

[59] S. Picozzi, A. Continenza, and A.J. Freeman: Phys. Rev. B 66, 094421 (2002)

[60] S. Picozzi, A. Continenza, and A.J. Freeman, IEEE Trans. Magn. 38, 2895 (2002)

[61] I. Galanakis, S. Ostanin, M. Alouani, H. Dreyssé, and J.M. Wills,: Phys. Rev. B 61, 4093 (2000)

[62] M.C. Kautzky, F.B. Mancoff, J.-F. Bobo, B.M. Clemens, et al.: J. Appl. Phys. 81, 4026 (1997)

[63] K. Inomata, S. Okamura, R. Goto, and N. Tezuka: Jap. J. Appl. Phys. Part 2-Lett. 42, L419 (2003)

[64] G.A. de Wijs and R.A. deGroot: Phys. Rev. B 64, 020402 (2001)

[65] K. Westerholt, U. Geiersbach, and A. Bergmann: J. Magn. Magn. Mater. 257, 239 (2003)

[66] U. Geiersbach, A. Bergmann, and K. Westerholt: Thin Sol. Films 425, 226 (2003)

[67] U. Geiersbach, A. Bergmann, and K. Westerholt,: J. Magn. Magn. Mater. 240, 546 (2002) 
[68] H. Bach, K. Westerholt, and U. Geiersbach: J. Cryst. Growth 237, 2046 (2002)

[69] A. Bergmann, J. Grabis, V. Leiner, M. Wolf, H. Zabel, and K. Westerholt: Superlattices and Microstructures 34, 137 (2003)

[70] A. Nefedov, J. Grabis, A. Bergmann, K. Westerholt, and H. Zabel: Physica B 345, 250 (2004)

[71] J. Grabis, A. Bergmann, A. Nefedov, K. Westerholt, and H. Zabel: Phys. Rev. B submitted

[72] P. Bruno: Phys. Rev. B 52, 411 (1995)

[73] M. Goto, T. Kamimori, H. Tange, K. Kitao, S. Tomioshi, K. Ooyama, and Y. Yamaguchi: J. Magn. Magn. Mater. 140-145, 277 (1995)

[74] J. Stöhr: J. Magn. Magn. Mater. 200, 470 (1999)

[75] J. Grabis and A. Nefedov, and H. Zabel: Rev. Sci. Instr. 74, 4048 (2003)

[76] H.A. Dürr, G. van der Laan, D. Spanke, F.U. Hillebrecht, and N.B. Brookes: Phys. Rev. B 56, 8156 (1997)

[77] J. Nogues, and I.K. Schuller: J. Magn. Magn. Mater. 192, 203 (1999)

[78] P. Miltenyi, M. Gierlings, J. Keller, B. Beschoten, G. Güntherodt, U. Novak, and K.D. Usadel: Phys. Rev. Lett. 84, 4224 (2000)

[79] A.P. Malozemoff: Phys. Rev. B 35, 3679 (1987)

[80] D. Mauri, H.C. Siegmann, P.S. Bagus, and E. Kay: J. Appl. Phys. 62, 3047 (1987)

[81] S.J. Blundell and J.A.C. Bland: Phys. Rev. B 46, 3391 (1992)

[82] H. Zabel: Physica B 198, 156 (1994)

[83] H. Zabel, and K. Theis-Bröhl: J. Phys.: Condens. Matter 15, S505 (2003)

[84] K. Theis-Bröhl, V. Leiner, A. Westphalen, and H. Zabel: Physica B 345, 161 (2004)

[85] B.D. Culity: Introduction to Magnetic Materials, (Addison-Wesley, 1972)

[86] D. Altbir, M. Kiwi, Ramírez, and I.K. Schuller: J. Magn. Magn. Mater. 149, L246 (1995)

[87] L. Neél: C.R. Acad. Sci. 255, 1545 (1962)

[88] D. Wang, J.M. Daughton, Z. Qian, C. Nordman, M. Tondra, and A. Pohm: J. Appl. Phys. 93, 8558 (2003) 
[89] J.A. Borchers, J.A. Dura, J. Unguris, D. Tulchinsky, M.H. Kelley, C.F. Maijkrzak et al.: Phys. Rev. Lett. 82, 2796 (1999)

[90] Ch. Rehm, D. Nagengast, F. Klose, H. Maletta, and A. Weidinger: Europhys. Lett. 38, 61 (1997)

[91] Melvin D.Daybell: The Kondo Problem, in: Magnetism V, ed. by G.T. Rado and H. Suhl (Academic Press, New York and London, 1973) p. 121

[92] P.A. Lee and T.V. Ramakrishnan: Rev. Mod. Phys. 57, 287 (1985)

[93] B.L. Altshuler and A.G. Aronov: Sov. Phys. JETP 50, 968 (1979)

[94] W.L. McMillan and Jack Mochel: Phys. Rev. B 46, 556 (1981) 